\title{
Hat die Bistumspresse noch eine Zukunft?
}

Die Artikel des voraufgegangenen Heftes 2/96 waren den "Chancen der Bistumspresse" gewidmet, wie sie sich aus der Allensbach-Studie 1995 ergeben. In diesem Heft stellen wir einige Beiträge zur Diskussion, die sich kritisch mit den Ergebnissen der Umfrage und den Problemen der Bistumszeitungen im Zusammenhang mit dem Gesamtproblem kirchlicher Kommunikation befassen.

\section{Ausstieg aus dem Fahrstuhl Allensbach, Kepplinger und die Kirchenzeitungen}

\author{
von Hartmut Paeffgen
}

Sind die Kirchenzeitungen zu retten? Diese Frage ist keine Provokation, sondern stellt sich fast zwangsläufig angesichts der kontinuierlich fallenden Abonnenten- und Leserzahlen einer Publikationsform, die der Hamburger Medienwissenschaftler und Journalistik-Professor Dr. Dieter Roß beim Treffen katholischer und evangelischer Journalisten und Medienschaffenden im Juni 1996 in der Hansestadt "einen Anachronismus" nannte. Aber auch wenn die Zeit der "Gesinnungspresse" schon lange vorbei sein sollte: Noch immer finden - trotz dramatischer Einbußen insbesondere in den letzten Jahren - 1,2 Millionen Kirchenzeitungs-Exemplare ihre Abnehmer und Leserschaft. Hinter diesen Zahlen steckt nicht nur ein Auftrag, sondern auch ein Stück Ermutigung und Ansporn für Herausgeber, Verlage und Redaktionen.

Also unverdrossen weiter so? Wer dies schon bisher nicht für möglich hielt, sondern eher für eine Einbahnstraße in der Aussichtslosigkeit, darf sich durch eine Untersuchung bestärkt fühlen, die das Institut für Demoskopie Allensbach im Auftrag der Medien-Dienstleistung Gsellschaft (MDG) in München unter dem Arbeitstitel "Chancen für die Bistumszeitung" durchführte. Das Ergebnis, auf einen kurzen Nenner gebracht: Die kirchliche Presse und insbesondere die Bistumsblätter haben eine Zukunft - aber nur, wenn sie ihr Profil und ihre Qualität verbessern, für neue Leserschichten attraktiv werden und im harten Marktwettbewerb bestehen!

Kaum waren erste Ergebnisse der Allensbacher Studie und Teile der begleitend von der MDG in Auftrag gegebenen Gestaltungs- und

Hartmut Paeffgen ist Chef vom Dienst bei den "Stuttgarter Nachrichten" und war beteiligt an den MDG-Seminaren zur Umsetzung der Allensbach-Untersuchung. 
Inhaltsanalyse von Professor Kepplinger vom Institut für Publizistik der Johannes Gutenberg Universität Mainz bekannt, gab es Kritik, Vorbehalte und Einwände. Das war nicht anders zu erwarten. Wer verabschiedet sich schon gerne von einer über Jahre gepflegten Meinung, wirft liebgewordene Vorstellungen (und Entschuldigungen) leichtherzig über Bord?

Hauptvorwürfe an die Adresse der "Untersucher": Der Kreis der Befragten sei zu klein gewesen, die Zahl der herangezogenen Bistumsblattleser reiche für gültige Aussagen nicht aus, die Ergebnisse seien nicht repräsentativ und wissenschaftlich fragwürdig, die Untersuchung und die Analyse berücksichtigten nicht oder nur zu wenig die speziellen Struktur-, Personal- und Sachzwänge für Verlage und Redaktionen.

Vielfach wohl bewußt negiert bzw. völlig außer acht gelassen wurde und wird bei dieser Kritik dreierlei:

Erstens die erfreuliche Tatsache, daß erstmals überhaupt eine so umfangreiche Untersuchung der Bistumszeitungen, die weit über die Erhebungen der Feldbefragung 1975 hinausreicht, vorgenommen und unter den unterschiedlichsten Aspekten ausgewertet wurde;

zweitens das in seiner Bedeutung offenbar unterschätzte Resultat, $\mathrm{da} \beta$ Herausgeber, Verlage und Redaktionen im Hinblick auf Leserstrukturen, Inhalte, Erwartungen und Leseverhalten nicht mehr allein auf Annahmen und Vermutungen angewiesen sind, sondern tatsächlich etwas "wissen“;

drittens die da und dort bereits begonnene Aufarbeitung und Umsetzung der Untersuchung. Die intensiven Seminare mit immerhin 18 Verlagen und Redaktionen von Bistumszeitungen, in denen die Ergebnisse erwartungsgemäß heftig diskutiert wurden, waren für nicht wenige Initialzündung und Anlaß zu einem "Ruck nach vorn". Aus dem Gesamtprojekt wird auch ein "Stylebook" als Hilfe für den Redaktionsalltag entstehen. $\mathrm{Ob}$ und wie die darin gebündelten Erfahrungen und Empfehlungen umgesetzt werden, davon hängt viel ab.

Es beeinträchtigt m.E. nur unwesentlich den Wert und Nutzen der Allensbacher Studie, daß die Untersuchung auch einige Schwächen und "weißen Stellen" aufweist. So bleibt beispielsweise die Frage, wie und in welchem Umfang die Bistumszeitungen auf regionale Besonderheiten, Erwartungen und Mentalitätsunterschiede Rücksicht nehmen müssen, weitgehend unbeantwortet. Denkt, empfindet, interessiert die Leserin in Bad Tölz dasselbe wie die in Düsseldorf oder Bautzen? Das ist kaum anzunehmen.

$\mathrm{Zu}$ wenig ins Blickfeld gerückt wurde in der Untersuchung auch das nationale wie jeweils regionale Medienumfeld der Bistumszeitungen. In einem Fall berichten die Tageszeitung, Hörfunk und Fernsehen 
kaum über kirchliche Themen, in anderen Fällen und andernorts informieren gleich mehrere Medien regelmäßig, umfassend und aktuell über Ereignisse in Bistum und Weltkirche. Wie wirkt sich das auf die Bistumszeitung aus, wie groß sind jeweils ihre Chancen?

So betrachtet, bleibt das von Allensbach ermittelte "psychologische Interessenpotential von 31 Prozent" für eine kirchliche oder christliche Zeitschrift nichts weiter als eine statistische Größe, mit der allein wenig anzufangen ist. Überhaupt: Wie wüßte eine solche Zeitung oder Zeitschrift aussehen, welche Themen müßte sie anpacken, um beispielsweise jüngere Generationen anzusprechen und damit den Ausstieg aus dem Fahrstuhl nach unten zu schaffen?

Bei einer nachwachsenden Generation, für die Multimedia kein Schreckgespenst ist, tut sich die Kirche, tun sich aber insbesondere die Printmedien schwer wie nie zuvor. Wenn sie "die Jüngeren" erreichen wollen, werden sie auf andere, neue Kommunikationswege im elektronischen Bereich sicher nicht verzichten können. Das aber wird zusätzlich Kosten in erheblicher Höhe verursachen und den Verteilungskampf um die ohnehin knapper werdenden Mittel weiter verschärfen.

Der Leser - ein unbekanntes Wesen? Dank der AllensbachUntersuchung stochern die Verlage und Redaktionen nicht mehr nur im Nebel der Vermutungen. Alter, soziale Stellung, inhaltliche Erwartungen, Leseverhalten und Lesedauer wurden abgefragt und statistisch erhoben.

Das schafft freilich nicht das Problem aus der Welt, daß viele Bistumszeitungen oft nicht einmal den Namen ihrer Leser(innen) kennen, geschweige denn über Adressenlisten verfügen. Wie unter solchen Bedingungen in Kontakt kommen, gemeinsame Aktionen planen, Leser-Blatt-Bindung vertiefen, Leserwerbung betreiben? Dieses Defizit zu beseitigen, verdient höchste Priorität.

Allensbach und Kepplinger sind sich einig in der Empfehlung, das Augenmerk bei der Verbesserung der Kirchenzeitungen auf die sogenannten Kernleser und auf die "nachwachsende Generation der etwa ab 40jährigen Katholiken“ zu richten. Begründung: Schon 1975 hätte Michael Schmolke auf die starke Überalterung der Kirchenzeitungsleser hingewiesen. Heute gehörten "die verbliebenen bistumszeitungstreuen Kernleser in noch höherem Anteil dem Alterssegment der 60 jährigen und älteren an". An anderer Stelle sagt die Allensbacher Untersuchung, daß sich "der Abonnentenstamm noch stärker als vor 20 Jahren auf den engeren Kreis der älteren, überwiegend weiblichen Kirchentreuen reduziert" hat.

Auch wenn die Grundtendenz richtig sein dürfte, sollten bei solchen Alterserhebungen keine vorschnellen (und damit leicht falschen) 
Schlüsse und Konsequenzen gezogen werden. Ich erinnere mich, daß der Bundesverband der Deutschen Zeitungsverleger vor mehr als 20 Jahren in allen seinen Verlautbarungen und Publikationen von der "Überalterung der Zeitungsleser" und einer immer größeren Rentnermehrheit unter den Zeitungsabonnenten sprach. Die Uberraschung war groß, als eine Umfrage unter mehreren tausend, mit Computerhilfe aus der Abonnentenliste ausgewählten Leserinnen und Lesern einer großen deutschen Tageszeitung diese totale Fehleinschätzung und damit auch manches Marketingkonzept vom Tisch fegte.

Die Printmedien müssen heute um jeden Abonnenten ringen. Ausnahmslos. Auch und besonders die Kirchenzeitungen. Gewinnen kann hier nur, wer professionell arbeitet und seinen Kunden ein Qualitätsprodukt liefert. Was Allensbach und Kepplinger zu Qualität, Erscheinungsbild und Anmutung der meisten Bistumsblätter sagen, mündet für Pessimisten in der Feststellung: Lieblos, freudlos, mutlos, hoffnungslos. Tatsächlich findet sich in den Zeitungen mehr brave Pflichtschuldigkeit als lustvolle Kreativität, viel Nachrichten-Einerlei und wenig spannend-unterhaltsamer Lesestoff.

Nachrichtenforum? Davon sind die meisten Blätter noch ebenso weit entfernt wie von der wirklichen Nähe $\mathrm{zu}$ ihren Lesern und zu deren Leben, Denken und Fühlen. Das gilt nicht weniger für die Mehrzahl der säkularen Zeitungen. Auch sie müssen gewaltig umdenken und sich mehr Mühe geben, wollen sie ihre alten Leser halten und neue gewinnen.

Das von den Redaktionen oft zu hörende Argument, daß sie für eine bessere und lesernähere Zeitung zu wenig Personal hätten, kann nicht gelten. Ideenreichtum, Phantasie, Kreativität und Meinungsfreudigkeit sind keine Frage der Personalstärke. Allerdings: Inhaltliche Verbesserungen und hohe journalistische Professionalität sind nicht kostenlos zu haben, erzwingen Einschnitte und Prioritäten, erfordern neue Formen der Arbeitsteilung und Kooperation über bisherige Bistumsgrenzen hinweg.

Ich habe mir angewöhnt, beim nachmittäglichen Blick über die Themen in der Tageszeitung, für die ich arbeite, bewußt in die Rolle eines Lesers zu schlüpfen: Interessiert mich, was ich da finde? Muß/will ich das wissen? Habe ich einen Nutzen davon?

Dieses "Leser-spielen" ist ein unerhört ernüchternder Vorgang und hilfreich dazu. Er zwingt nämlich zum ständigen Nachdenken über die entscheidende Frage: Wie informativ im Vergleich zu anderen (und häufig aktuelleren) Medien, wie verständlich und verläßlich, wie hilfreich, wie unterhaltsam, wie unverzichtbar ist meine Zeitung für die Leser? Bei der Durchsicht der kirchlichen Presse zwingt sich nicht der Eindruck auf, daß schon viele Redaktionen und Verlage sich regelmäßig und konsequent diese inzwischen überlebenswichtigen Fragen stellen. 
Was bleibt zu hoffen? Daß die Kirchenzeitungen nicht nur an Profil, Attraktivität und Ansehen gewinnen, sondern auch einen Weg finden, wie diese "Botschaft" zu den Lesern gelangt. Denn was nutzt das bestgemachte Blatt, wenn dies am Ende doch nur eine Minderheit registriert?

Wer im lauten Mediengetöse gehört werden will, muß tüchtig auf die Pauke hauen. Es spricht einiges für die Prognose: Je geballter die Kräfte, desto größer die Chancen, daß es mit den Kirchenzeitungen zumindest nicht weiter abwärts geht. Das wär' doch schon was!

\section{Praxisnahe Anregungen MDG als Schrittmacher der Bistumspresse}

von Josef Dewald

Längst wissen es alle und zu Genüge: Mit der Auflage der Bistumspresse in der Bundesrepublik geht es schier unaufhaltsam abwärts. Und das schon bald dreißig Jahre. Sie zählt zwar immer noch 1,2 Millionen meist ältere - Bezieherinnen/Bezieher, mehr als doppelt so viele waren es aber zu Beginn der sechziger Jahre. Die Zufriedenheit mit ihrem inhaltlichen Angebot - überwiegend Nachrichten, Berichte, Kommentare, Bildreportagen, geistliche Betrachtungen, Erzählungen - und ihrer zumeist noch etwas unauffälligen Gestaltung hält sich bei den Katholiken insgesamt in Grenzen. Die vielen Anstrengungen in den beiden letzten Jahrzehnten, die Auflage wenigstens zu stabilisieren und das redaktionelle Angebot zeitgerecht $\mathrm{zu}$ verbessern/zu verändern, führten bis heute nicht zum erwarteten Erfolg. Zu schwer tragen die Bistumsblätter noch immer an der Last ihrer Vergangenheit und den hausgemachten Schwächen. Sie kommen weithin, wenn überhaupt, nur mühsam von dem Image los, das „Blatt der Oma“ zu sein - und die älteren Herrschaften sehen es weiterhin gerne als "unser Blatt" an. Zu lange auch sind die Blätter ohne hohen journalistischen Anspruch gemacht worden, wurden sie verstanden als "publizistischer Herrgottswinkel" im braven katholischen Haus. (Man verstehe das bitte nicht als schnöde Kritik an Vorgängern in früherer, vorkonziliarer Zeit. Es soll nur dem besseren Verständnis der heutigen mißlichen Lage dienen.)

Josef Dewald ist seit 1969 Chefredakteur des Konradsblattes, der Bistumszeitung für das Erzbistum Freiburg. 
Nun soll dies unbedingt und mit kompetenter Beratung der Verlage und Redaktionen anders werden. Im Auftrag der kirchlichen Medien-Dienstleistung Gesellschaft (MDG) in München hat 1994/95 das Allensbacher Institut für Demoskopie eine gründliche Feldbefragung und umfangreiche Situationsanalyse der Bistumspresse erstellt. Und der Mainzer Publizistik-Wissenschaftler Hans Mathias Kepplinger hat parallel dazu eine vergleichende Inhaltsanalyse vorgenommen, ebenfalls im Auftrag der MDG. Zu deren Credo gehört es, daß der Bistumspresse bei zeitbedingten Anpassungen eine Zukunft gewiß ist. Kepplingers Analyse und mehr noch die von ihm daraus abgeleiteten Empfehlungen sind da und dort in den Redaktionen jedoch als wenig wirklichkeitsnah und hilfreich empfunden worden; mal abgesehen von der Feststellung, daß bei den Kirchenzeitungen die defensiven Aussagen über Kirche und Glauben überwiegen würden und die Lebenswelt der Bezieher/Leser zu wenig Beachtung finde. Anders die Einschätzung der Allensbach-Daten. Sie vermitteln insgesamt ein unvoreingenommenes, unverzerrtes Bild.

Damit allein ist freilich noch wenig erreicht, folgt der Diagnose nicht eine angemessene Therapie. Der ersten Feldbefragung zur Bistumspresse in den siebziger Jahren, die selbst schon etwas fragwürdig war, kam recht wenig hinterher, was auf einen ernsten Willen bei den Bistumszeitungen schließen ließ, mutig und zuversichtlich Neues zu erproben. Es wurde mit mancherlei Rechtfertigung in gewohnter Manier weitergemacht. Und so wäre es wohl aufs Ganze gesehen auch diesmal geschehen, zumal die Befunde weder eine einvernehmliche Einschätzung der Bistumspresse noch einheitliche Erwartungen vermitteln. Erstaunlich dabei: die Bezieher, meist ältere Jahrgänge, wie schon angedeutet, sind mit ihrem jeweiligen Bistumsblatt eher zufrieden. Kritik, Geringschätzung, Ablehnung kommt dagegen überwiegend von Jüngeren. Auch sind - um bei dem gängigen Klischee zu bleiben Konservative eher zufrieden als Progressive. Herbe Konsequenz: die Bistumszeitung, die mit den Jüngeren und Erneuerungswilligen fortbestehen, nicht mit den Älteren und den Beharrenden den Weg alles Irdischen gehen will, muß zwangsläufig Konflikte mit ihren zumeist treuen Abonnenten riskieren, Angriffe auf ihre Lesegewohnheiten wagen, wie behutsam und kalkuliert auch immer. Und sie muß als Vermittlerin etwa zwischen Rechten und Linken, als ein unparteiisches Dialogforum um Vertrauen werben, dem Aufruf zur Mitsprache der Gläubigen gerecht werden, sich auch als "Hörrohr des Bischofs" bewähren. Sie muß ferner erreichen, daß sie von den Hauptamtlichen in der Kirche endlich ernst- und angenommen wird. Viele, zu viele von ihnen - Priester wie Laien - erwarten immer wieder etwas über sich darin, kaum aber etwas für sich. Und bei den Ehrenamtlichen ist dies wenig anders.

Ein hartes Brot, gewiß. Aber auch da war und ist die MDG ihrem Auftrag gemäß diesmal beratend und helfend zur Stelle. So hatte sie als Beispiel seien sie erwähnt - in der zweiten Hälfte des vergangenen Jahres zu Seminaren zur Umsetzung der Feldbefragung und der 
Inhaltsanalyse eingeladen, und dabei, das sei gleich vorweg gesagt, Gelegenheit gegeben, in Werkstatt-Gesprächen Erfahrungen auszutauschen und reichlich praxisnahe Anregungen zu erhalten; Skepsis, im Vorfeld auch von mir geäußert, hat sich als unbegründet und ungerechtfertigt erwiesen.

Was bei einem dieser Seminare im Oktober 1995 in München der Kollege Hartmut Paeffgen von den „Stuttgarter Nachrichten“ als seinen Befund der Bistumspresse vortrug, war für Insider alles andere als aufregend neu, aber es war in seiner Bündelung in heilsamer Weise ernüchternd und im guten Sinne des Wortes anstößig. Die Bistumsblätter, so der Kollege von der Tagespresse mit geschultem Blick, seien zu problemlastig und die Beiträge oft zu lang, inhaltlich auch falsch gewichtet. Es würden zu viele Nachrichten weitergegeben und $\mathrm{zu}$ wenig ergänzende Hintergrund-Information geliefert. Auch stellte er einen Mangel an hinreichend qualifizierter Orientierung und Kommentierung fest. Graphiken, Schaubilder würden überwiegend fehlen, Bilder in ihrem Eigengewicht unterschätzt. Ebenso fehle ein Service-Teil sowie Lebensberatung und -hilfe, die den Namen verdiene. Den Redaktionen hielt Paeffgen vor, sie würden zu wenig selbst erarbeiten und ihre Leistung sei insgesamt unbefriedigend. Er räumte jedoch ein, daß dies am wenigsten ihre Schuld sei, da sie noch immer nicht ausreichend besetzt seien. Anzumerken ist dazu, daß die Redaktionen heute zwar zumeist personell wie finanziell besser ausgestattet sind als je zuvor, daß aber zugleich die Leistungen, die Woche für Woche von ihnen erwartet werden - beim Redigieren, Recherchieren, Kommentieren, Organisieren, Koordinieren, Wahrnehmen von Terminen - die Anforderungen früherer Jahre um ein Vielfaches übersteigen. Fern die Zeit, wo weit überwiegend Unterhaltung und Erbauung die Bistumspresse kennzeichneten, ihr "Gütesiegel " waren, wo im "endlosen Chor" Bilder der Heiligen die Titelseiten füllten, und dies bei hoher, stabiler Auflage und nicht minder stabilem Verkaufserlös.

Hartmut Paeffgen ging es überhaupt am wenigsten um Kritik im üblichen Verständnis des Wortes, also um Kritik als Widerspruch. Ihm war daran gelegen, die Augen für die Wirklichkeit weiter öffnen zu helfen und Anregungen zu vermitteln für eine zeitgerechte Darbietung der Bistumsblätter. Seine Tips entsprechen dem erwähnten Befund: Ergänzende, vertiefende Hintergrund-Informationen sind wichtiger als aktuelle Nachrichten, wie sie eh schon die Tagespresse und die elektronischen Medien bringen; wichtig sind ebenso Stellungnahmen aus kirchlicher Sicht zum aktuellen Geschehen; wo immer möglich, ist bei Berichten und Reportagen, soweit sie nicht aus dem eigenen Bistum sind, ein ergänzender regionaler/diözesaner Bezug herzustellen; alle Themen sind ausgewogen $\mathrm{zu}$ behandeln, nicht einseitig, nicht parteiisch; einfühlsame Glaubenshilfe ist zu leisten, nicht apologetische Glaubensverkündigung; fachkundige Lebenshilfe darf in keinem Heft fehlen, ebenso wenig im Alltag brauchbare, verwertbare Informationen in Form einer Dienstleistung; Fernsehen und Hörfunk sind kommentierend zu 
begleiten; unterhaltende Beiträge sind unverzichtbar, haben jedoch dem Kriterium "Lesespaß $\beta^{\prime}$ zu genügen. Zukunft gibt Paeffgen der Bistumspresse, soweit sie sich positiver, mehr dem Leben zugewandt als bisher, zuversichtlicher präsentiert, wenn sie in Gestaltung und Sprache dem Leser mehr entgegenkommt.

Es ist keine Frage, daß das redaktionelle Profil der Bistumsblätter derart fortzuentwickeln ist, soll es auf die Höhe der Zeit gelangen, den Erwartungen jüngerer, kritischer, dialogwilliger Leser gerecht werden. Und es ist hierbei auch nicht von vornherein und generell eine Abwehrhaltung aller Älteren $\mathrm{zu}$ befürchten; Medienpädagogik in eigener Sache, etwa in Form eines "Tagebuchs der Redaktion", in dem Fragen zu Veränderungen/Neuerungen eine plausible Antwort finden, ist dabei hilfreich. Aber wenn die Lebenshilfe und Lebensberatung konkret werden soll, zum Beispiel bei der Frage, ob es moralisch vertretbar ist, daß junge Leute ohne Trauschein zusammenleben und ob es kirchlich zu rechtfertigen ist, daß sie dann auch beim gelegentlichen Sonntagsgottesdienst zur Kommunion gehen, dann wird es nach allen Erfahrungen schwierig. Da macht sich leicht unglaubwürdig, wer redlich und verantwortlich vermitteln will, ohne von der Lehre der Kirche abzuweichen. Das gilt insgesamt bei schwierigen theologischen, pastoralen oder kirchenrechtlichen Fragen. Man denke etwa an Reizthemen wie Jungfrauengeburt, Auferstehung/ Auferweckung Jesu oder den kirchlichen Umgang mit geschiedenen Wiederverheirateten. Auch die Ökumene wirft Fragen auf, die ohne weiteres keine einvernehmlichen Antworten finden. Der gegenwärtige Problemstau in der Kirche auf der einen und das Festhalten am Überlieferten ist aufs Ganze gesehen schier übergroß. Ausweichen, den Kopf in den Sand stecken, ist freilich keine Lösung. Wo aber sind die Autoren/die Autorinnen in genügender Zahl, die umsichtig und wissenschaftlich kompetent genug für die Bistumspresse gegenwartsnahe, maßgerechte und zu dem verständliche Beiträge schreiben? Die Suche nach ihnen gleicht fast der Suche nach der Stecknadel im Heuhaufen.

Mit anderen Worten: Die Lage der Bistumspresse ist viel schwieriger, problematischer als sich von außen zeigt, als sich in allgemeinen Situations- und Inhaltsanalysen darstellen läßt und als ein noch so hilfreiches Tagesseminar zur Sprache bringen kann. Dennoch oder gerade deshalb ist es gut, daß jetzt in ersten Schritten und mit ausgewiesenen Fachleuten wirklich ernsthaft versucht wird, Neuland zu gewinnen. $A$ und $O$ in dieser Lage eines ungewissen, gewagten Ubergangs muß es sein, auch wenn es sich als Quadratur des Kreises erweist, das Vertrauen der Älteren, der Rückwärtsgewandten, der fest im Glauben verwurzelten, der bis jetzt mit den Blättern einigermaßen Zufriedenen zu erhalten, weiter zu rechtfertigen und das derer mehr und mehr zu gewinnen, die ihnen mit Vorbehalten unterschiedlicher Art begegnen. Sie müssen sich für den einen weiterhin und für die anderen in neuer Weise als vertrauenswürdig erweisen. Doch nur soweit sich die Bistumspresse dieser "Quadratur des Kreises" stellt und 
die ihr gelingt, trägt sie ihre Kennzeichnung, Zeitung der Ortskirche zu sein, weiter zu Recht. Mit sogenannten zielgruppenspezfischen SonderAngeboten alleine ist es allerdings nicht getan, schon deshalb nicht, weil Ältere sich gegen eine ungewohnte, jugendgemäße Sprache rasch wehren und Junge unumwunden wissen lassen, daß sie eine Sprache, die Älteren vertraut ist, zum Gähnen finden.

Die Last der Bistumspresse besteht also mehr denn je darin, als Leserschaft $\mathrm{zu}$ vereinen, was eher auseinanderstrebt, sich voneinander abzugrenzen sucht, was sich mit Argwohn begegnet, was in verschiedenen geistigen und religiösen Welten lebt. Aber bei diesen Lesergruppen in ihrer legitimen Vielfalt füreinander Interesse zu wecken, aufeinander neugierig zu machen, mehr Dialogbereitschaft und -fähigkeit zu entwickeln, ist eine ihrer wesentlichen Aufgaben, und es ist wohl ihre einzige Chance, mit einer Auflage, die den Aufwand lohnt, als "Gemeindeblatt" zu überleben. Wie hoch diese Chance zu veranschlagen ist, läßt sich gegenwärtig schwer abschätzen - nicht zuletzt in Anbetracht der weiter rückläufigen und sich weit auffächernden kirchlichen Religiosität und Kirchlichkeit überhaupt. Noch kann von einer Trendwende zum Besseren keine Rede sein. Eine Voraussetzung für ihr Gelingen ist auch, daß sich Herausgeber, Verleger und Redaktion nicht auseinanderdividieren lassen. Konkret schließt dies mit ein, daß Herausgeber auch Themen, Darlegungen und Denkungsarten zulassen, die sie nicht unbedingt erfreuen; daß Verleger verstärkt zu unternehmerischem Risiko und neuen Wegen in der Werbung von Beziehern und der Imagepflege bereit sind; daß sich Redaktionen ebenso durch Wagemut wie durch Umsicht auszeichnen, durch einen geschärften Blick wie durch Augenmaß.

Wo es um die Zukunft der Bistumspresse geht, fällt - hauptsächlich von Beratern und kirchlichen Medienverantwortlichen in überdiözesanen Gremien - häufig das Stichwort "Kooperation". Auf den Spuren von Allensbach und Prof. Kepplinger sieht selbst der Vorsitzende der Publizistischen Kommission der Deutschen Bischofskonferenz, der Trierer Bischof Hermann Josef Spital, diesbezüglich „Handlungsbedarf . Verstanden wird darunter allerdings in einer wenig nachvollziehbaren Engführung vor allem die redaktions-technische Kooperation, also die gemeinsame Produktion von bestimmten Seiten oder eines bestimmten Teils der Zeitungen in regionalem Verbund. Ihre Befürworter erwarten sich von ihr eine Verbesserung der Qualität durch Bündelung der redaktionellen Kräfte bei gleichzeitiger Senkung der Produktionskosten - demnach ausschließlich Günstiges. Was ihnen vorschwebt, ist eine Zusammenlegung von Blättern bis hin zur Einrichtung von Zentralredaktionen. In den einzelnen diözesanen Redaktionen soll dann nur noch wahrgenommen werden, was das dortigen Geschehen betrifft.

Am grünen Tisch klingt da manches plausibel und zustimmungsfähig, was sich im konkreten Alltag aber eher als problematisch erweist. Bereits vorhandene Kooperationen solcher Art sind allenfalls unter der 
Voraussetzung drückender Kosten durch zu geringe Auflagen ein Modell auf absehbare Zeit. Vielleicht sind sie aber auch nur eine Möglichkeit, auf Dauer unumgängliche Rückzugsgefechte der kirchlichen Presse in einem milden Licht erscheinen zu lassen, eine Art "konservative Resignation".

Sinnvoll wäre in jedem Fall zunächst einmal, die redaktionellen Kooperationen bewußt als solche in den Blick zu nehmen, die seit Jahrzehnten vorhanden sind und sich Woche für Woche bewähren: Namentlich die Kooperation mit der Katholischen Nachrichten-Agentur, mit KNA-Bild und mit dem Kölner Medieninstitut. Alle drei Einrichtungen leisten für die Bistumspresse unverzichtbare und qualitativ hochwertige Zubringer-Dienste, die über das gewöhnliche Miteinander von Redaktionen und Agenturen weit hinausgehen. Und diese Dienste sind weiter ausbaufähig im Sinne neuer Anforderungen an die Bistumspresse, etwa in den Bereichen Lebensberatung und -hilfe, Dienstleistungen, Hintergrund-Informationen, Darstellung theologischer Streitfragen. Da die einzelnen Blätter hierbei keine fertigen Seiten zu übernehmen haben, läßt sich zudem in hohem Maß einer diözesanen Ergänzung und Anreicherung von Texten und Bildern, wie sie Hartmut Paeffgen zu Recht für wichtig und zukunftsweisend hält, in ansprechender Weise Rechnung tragen.

Was ebenfalls wert ist, weiter verbessert $\mathrm{zu}$ werden, das ist die redaktionelle Kooperation verschiedener Blätter miteinander. Da gibt es bis heute leider nur die Zusammenarbeit der Blätter Aachen, Trier, Mainz/Limburg/Fulda, Freiburg bei der gemeinsamen wöchentlichen Rubrik "Anfrage“. Sie gibt, gemeinsam von den Theologen dieser Redaktionen verantwortet, in knapper Form Auskunft auf Fragen von Lesern/Leserinnen aus den Bereichen Theologie, Pastoral, Liturgie, Kirchengeschichte, Moral, Kirchenrecht. Und da gibt es noch die gemeinsame Rubrik "Lebenshilfe" ebenfalls dieser Blätter mit Ausnahme von Aachen und dafür noch mit Speyer. Sie bringt vierzehntägig Beiträge der Psychologin und Pädagogin Dr. Marle Kallen. Es ist wenig verständlich, daß diese Beispiele nicht längst bei anderen Schule gemacht haben.

Zumindest soweit wirtschaftliche Zwänge es nicht unumgänglich machen, gemeinsam fertige Seiten zu bringen, sollten erst einmal diese Möglichkeiten redaktioneller Kooperation ausgiebiger genutzt werden. Mit ihnen ist eine Verbesserung des redaktionellen Angebots $\mathrm{zu}$ erreichen, ohne den Blättern ihre diözesane Verankerung und „Einfärbung" zu nehmen. Ob sich mit ihnen die Zukunft gewinnen läßt, ist freilich auch nicht gewährleistet. 


\title{
Für Gott und die Welt - Die eigene Sprachlosigkeit überwinden \\ Widrigkeiten und Chancen für die Bistumspresse aus der Sicht eines dafür Verantwortlichen
}

\author{
von Roland Juchem
}

Studiert habe ich Theologie und Pädagogik, ausgebildet bin ich als Journalist. Als solcher arbeite ich in der sogenannten Kirchengebietspresse. Andere sprechen vom "Kirchenblättchen". Fünf Jahre war ich als Redakteur bei "Kirche und Leben" in Münster tätig, seit knapp einem Jahr bei der "Kirchenzeitung für das Bistum Aachen“. Auf die Schwierigkeiten meines Berufes angesprochen antworte ich gern mit dem „Spagat": "Als Blatt für die Katholiken des Bistums müssen wir für unsere Leserschaft ständig einen Spagat hinlegen."

Das mit dem Spagat ist mir schon sehr bald aufgefallen, kurz nachdem ich als frisch diplomierter Theologe die bunte Welt des Katholizismus so erlebte, wie sie sich in Leserzuschriften und Telefonanrufen bei der Redaktion kundtut. Von Pax-Christi-Demonstranten und feministischen Theologinnen über die KAB-Funktionäre und Kolpingbrüder bis hin zu den Lepra-Strickkreisen und WallfahrtsBruderschaften - für sie, allesamt Katholiken und Katholikinnen des Bistums, sollten wir ein Blatt machen? Ganz zu schweigen von den Pfarrern, Pastoralreferentinnen und Hauptabteilungsleitern des Generalvikariates. Sisyphos läßt grüßen. Da war ein Bischof als Herausgeber, den sich Kollegen aus der sogenannten säkularen Presse gerne als "Oberzensor" vorstellen, noch harmlos.

Gut, diese Kollegen haben ebenfalls eine bunt zusammengewürfelte Leserschaft. Aber sie arbeiten für eine Tageszeitung. Und die halten sich die meisten Leute, können sie als lokale Tageszeitung mangels Alternative auch kaum wechseln. Aber eine Wochenzeitung, das ist immer etwas Zusätzliches, etwas Besonderes, in Zeiten knapper Haushaltsgelder mitunter ein Luxus. Da möchte der Leser dann bitte etwas haben, das hundertprozentig auf seine Interessen paßt und ihm möglichst die Freude an seiner Meinung nicht verdirbt.

Ich bezweifle, daß es das katholische Milieu je gegeben hat. Katholiken in Deutschland sind ein buntes und vielfältiges Volk, dessen Mitglieder heute - wie der Rest der Gesellschaft - zudem etwas individueller geworden sind. Sie alle mit einem einzigen Blatt bedienen

Roland Juchem ist Redakteur bei der Kirchenzeitung für das Bistum Aachen. 
zu wollen, ist mehr als utopisch. Es gibt rund zwei Dutzend Computerzeitschriften, jede mit einem eigenen Schwerpunkt, Magazine für stehende, sitzende und liegende Surfer. Bei allem Respekt vor christlicher Widerständigkeit gegen den Zeitgeist - da machen wir keine Wochenzeitschrift für die Christen eines Bistums.

Jede bessere Erhebung der Leserschaft, zuletzt die von Allensbach und MDG von 1994/95, zieht dem eingangs erwähnten Spagat den Teppich unter den Füßen weg. Journalistisch engagierte Männer Mitte Dreißig machen ein Blatt für kirchlich interessierte Frauen ab sechzig. Zugegeben, das ist stark übertrieben, weist aber auf eines der wesentlichen Probleme der Bistumspresse hin. Die eingangs skizzierte Bandbreite katholischer Leserschaft wird von Herausgeber und Redaktion zwar gerne angepeilt, Anzeigen- und Vertriebsabteilung belehren sie aber meist eines besseren.

Es gibt noch andere Widrigkeiten, die die Chancen der Bistumspresse auf eine bessere Auflagenentwicklung erheblich mindern. Weil sie an anderer Stelle, auch in diesem Heft, ausführlicher beschrieben werden, genügen hier Stichworte:

1. Die Zahl der Menschen, die sich für kirchliche Themen interessiert, nimmt ab. Und nachdem die Kirchensäckel leer sind, wird mittelfristig auch die Zahl derjenigen sinken, die sich beruflicherseits für Kirchliches interessieren.

2. Die Beziehung oder gar Identifikation der Menschen mit Institutionen ist rapide gesunken. Und in den gegenwärtigen Formen von Herausgeberschaft werden Bistumszeitungen ihren Makel, offiziöses Blatt "der Kirche" zu sein, nicht ablegen können. Damit will ich nicht bestreiten, daß diese gegenwärtige Form der Herausgeberschaft (Bischof oder Bistum) für eine Gruppe von Katholiken ein klarer Vorteil, ein Kriterium für die Bistumszeitung ist.

3. Die ganz Alten unter der älteren Leserschaft sterben weg. Ob aber die jungen Alten als neue Abonnenten hinzugewonnen werden können, ist fraglich. Auch sie sind so plural wie das übrige Gottesvolk; und wenn sie bis dahin keine Beziehung zur Kirche gehabt haben, ist es schwer, sie für eine Zeitschrift zu begeistern, die ihnen bis dahin nichts hat bieten können.

4. Bei aller Renaissance, die das Lokale, die Region, die Heimat gerade im Medienbereich erfahren haben, die kirchliche Verwaltungsgröße Bistum profitiert leider nicht davon. Christen von heute engagieren sich - oft zeitlich begrenzt - für ihre Gemeinde, den Verband vor Ort oder aber in der weiten Welt, in Brasilien, Südindien oder Ghana. Aber auch das Interesse an Spiritualität, Menschenrechtsarbeit, Bewahrung der Schöpfung oder ähnlichem kann weitestgehend auf eine Identifikation mit dem Bistum, in dem man gerade lebt, verzichten. 
5. Wochen- und Fachzeitschriften - die Bistumszeitung ist wohl eine Mischung aus beidem - müssen, wie gesagt dem Bedürfnis und der Weltsicht des Lesers, der sie sich leistet, entsprechen. Nicht, daß er sich nicht hin und wieder mit anderen Meinungen und Lebensentwürfen konfrontiert sehen wollte; aber Aufmachung, Stil, Themenauswahl und Meinungsschwerpunkt sollten den eigenen Vorstellungen schon entsprechen. Hinzu kommen besondere Empfindlichkeiten in allen Fragen des Kirchlich-Religiösen. Da wird mit einer anderen Elle gemessen, als wenn's ums Hobby, den Lebensstil oder politische Fragen geht. Zusammen mit der Tatsache, daß Ansprüche, Erwartungen und Geschmäcker der Katholiken eines Bistums heute noch unterschiedlicher und individueller geworden sind, begrenzen diese Faktoren die Möglichkeiten einer Bistumszeitung noch mehr.

Bei der jüngsten gemeinsamen Jahrestagung von AKP und GEP in Hamburg stellte jemand in einer Arbeitsgruppe die Frage: Können Journalisten der Bistums- oder Kirchenzeitung gegen solche strukturellen Widrigkeiten überhaupt anschreiben? Sind nicht eher verlegerische Impulse gefragt? Vorstellbar wären ja, da das einzelne Bistum als Bezugsgröße nicht mehr so gefragt ist, zwei oder drei konkurrierende Kirchenzeitungen, die aber ein größeres Gebiet abdecken. Ein eher konservatives Blatt, ein eher liberal-progressives. Oder eines, das eher spirituell, und eines, das eher politisch ausgerichtet ist. Die einzelne Herausgeberschaft kann, muß aber nicht beim Bischof/Bistum liegen. In der Arbeitsgruppe war kein Verleger anwesend, sonst wären derartige Gedanken vielleicht weitergesponnen worden - oder sofort beendet gewesen.

Angesichts der erwähnten strukturellen Schwierigkeiten für die Bistumspresse ließe sich mit Blick auf ihre Chancen ebensogut fragen: Wäre es nicht besser, die Leute fänden in ihrer Lokalzeitung, was gerade vor Ort passiert, hielten sich für das Überregionale - je nach Geschmack und Anspruch - "Publik Forum", "Rheinischer Merkur", "Das Sonntagsblatt", den "L'Osservatore" oder die „Herderkorrespondenz", und jene, die theologisch noch spezieller informiert sein wollen, die eine oder andere theologische Fachzeitschrift?

Hinweise auf "strukturelle" Schwierigkeiten beinhalten oft sehr viel Richtiges, lenken aber ebenso oft $a b$ von der eigenen, der persönlichen Verantwortung. Die Leitfrage für die Arbeitsgruppe bei der AKP/GEP-Tagung lautete denn auch: "Tut die konfessionelle Presse ihre Pflicht?" Und von daher läßt sich zu den Chancen einer Bistumszeitung noch einiges mehr sagen.

$\mathrm{Da}$ wir in den Redaktionsstuben bistümlicher Wochenzeitungen nicht immer unsere journalistische Pflicht tun, daß sich etliches besser machen ließe, will ich gar nicht bestreiten. Dazu haben H.M. Kepplinger und S. Chr. Ehmig einiges Richtige gesagt. Wann aber hat Bistumspresse eine Chance? Dazu acht Überlegungen: 
1. Die Bistumspresse hat eine Chance, wenn sie sich als regional ausgerichtete Fachzeitschrift versteht. Als regionale Wochenzeitung muß sie weltkirchliche, allgemein-religiöse Themen lokal und persönlich verankern. Als Fachzeitschrift muß sie gezielt religiös-kirchliche Themen zur Sprache bringen; sprachlich aber nahezu voraussetzungslos schreiben. Das unterscheidet sie vielleicht von den üblichen Fachblättern. Wer gar nichts von Religion und Kirche weiß, greift nicht zur Bistumszeitung. $\mathrm{Da}$ aber der größte Teil auch der künftigen Leserschaft das Blatt nicht wegen fachtheologischer Fragen zur Hand nimmt, und zudem die Semiotik religiöser Sprache ("Was genau bezeichnen denn diese und jene Begriffe?") sehr unklar geworden ist, muß die Bistumszeitung voraussetzungslos fachkompetent schreiben.

2. Die Bistumszeitung muß jene Themen auswählen, die für Christen als Christen interessant sind und sie aus christlicher Perspektive behandeln. Das heißt weder, daß hinter jedem Absatz "das liebe Jesulein" hervorlugt, noch daß ein Thema nur deshalb ins Blatt kommt, weil irgendein Kleriker oder Funktionär eines katholischen Verbandes dazu etwas verlautbart hat.

3. Die Bistumszeitung muß konsequent für die Christen ihres Verbreitungsgebietes ein Forum bieten. Sie muß über alles berichten, was ist (mehr über das Geschehene als über das Gesagte), sofern es berichtenswert ist. Dafür gelten allein journalistische Kriterien. ${ }^{1}$ Die Bistumszeitung spiegelt die ganze Vielfalt des katholischen und ökumenisch ausgerichteten Lebens wider. Gegen die Trends zu gemeindlich-spirituellen "Kuschelgruppen", gegen Alleinvertretungsansprüche und Abkanzelungen ("Häretiker", "Kirchenfeind", "Neurotiker", "Rückständiger") sowie gegen die Insider-Codes soziologischer, psychologischer, politologischer, theologischer und frömmelnder Geistes-Clans bietet sie ein allgemeinverständliches Dialogforum. Sie ist geprägt durch einen ansprechenden, informativen, toleranten, aber auch profilierten Stil, auszuwählen, zu berichten und zu schreiben.

In einer Bistumszeitung erfahren die Leser, was Prof. Lüdemann über die Auferstehung Jesu oder Minister Kanther über Asylgesetzgebung sagen. Sie erfahren aber auch, was die Lehre der katholischen Kirche zur Auferstehung und die katholische Sozialethik zum Umgang mit Fremden sagt. Und sie erfahren, weshalb Lieschen Müller oder Herbert Meier sowohl mit Prüf. Lüdemann wie auch dem kirchlichen Lehramt ihre Schwierigkeiten haben.

4. Fragen nach Gott und der Welt gehören zu den spannendsten, die es

1 M.E. gibt es keinen eigenen christlichen Journalismus. Es gibt Christen, die als Journalisten arbeiten; und es gibt Journalisten, die über christliche, religiöse etc. Themen berichten. 
nach wie vor gibt. In ihnen muß sich die Bistumszeitung eine eigene Kompetenz erwerben und ständig unter Beweis stellen. Dabei ist "Gott und die Welt" durchaus auch im Sinn der gängigen Redewendung gemeint. Hochspezielle theologisch-philosophische Themen sind hier fehl am Platz. Nicht aber das, was Menschen in ihrem alltäglichen Leben bewegt, sei es tragisch oder unterhaltsam, wenn sie nach dem bißchen mehr an Substanz, Sinn und Tiefe fragen. Dabei leiden die Macher der Bistumszeitung jedoch ebenso unter der (scheinbaren) Abwesenheit Gottes, der religiösen Sprachlosigkeit und allgemeinen Bedeutungslosigkeit kirchlich-theologischer Rede.

Die eigene Verdauung und die eigene Spiritualität sind so ziemlich die letzten Tabuthemen, die unsere Medienwelt noch kennt. Es geht nicht darum, spirituelle Privatsphären ans Licht der Öffentlichkeit zu zerren. Aber es gibt eine große allgemeine Sprachlosigkeit in religiös-spirituellen Dingen. Zumal im katholischen Raum besteht eine große Scheu, darüber zu reden, selbst in kleinen Gruppen. Da können wir von unseren evangelischen Schwestern und Brüdern einiges lernen. Ihr Profil kann eine Bistumszeitung auch dadurch schärfen, daß sie dieser Sprachlosigkeit die Zunge zu lösen verhilft. Das ist ein großes Wagnis.

Wer in gedruckter Form öffentlich dem religiösen Suchen, Zweifeln und Hoffen der Menschen von heute sprachlichen Ausdruck verleihen möchte, läuft Gefahr, daß seine Leser ihn mißverstehen oder verdächtigen, seine Sätze gegen offizielle Lehrsätze auszuspielen suchen. Ebenso läuft er aber Gefahr, nichtssagend zu werden, alte und verbrauchte religiöse Begrifflichkeiten nur zu wiederholen oder seine Zeitung zur Kanzel zu machen. Im Grunde muß der Journalist, der religiös-spirituelle Dinge ins Blatt heben will, neue Sprachspiele erfinden und sie ständig auf ihre Tauglichkeit hin überprüfen.

5. Um zu überleben, muß die Bistumszeitung neue Leser gewinnen. Das kann sie aber nur, ohne die bisherigen, die sie in der bisherigen Form schätzen - sonst würden sie sie nicht beziehen -, zu verprellen. (Schon wieder ein Spagat!) Form und Inhalt, Sprache und Stil müssen den Themen angemessen sein, aber nicht von der übrigen (Presse-)Welt abgehoben.

Leser unterschiedlichen Alters unterscheiden sich nicht so sehr in dem, was sie thematisch interessiert, oder im intellektuellen Level. $\mathrm{Da}$ ist die Spannbreite innerhalb einer Altersgruppe schon genügend groß. Es ist allenfalls die Art der Aufmachung, die den Moden und damit dem Geschmack der Generationen unterliegt. Von Form, Inhalt und redaktionellem Auftreten her kann und muß die Bistumszeitung noch etliches von ihrem Negativ-Image als „Kirchenblättchen“ ablegen. Wenn sie zudem Fragen aufgreift, die tatsächlich 
gestellt werden, und über Dinge berichtet, die nicht nur um der Eitelkeit der jeweiligen "Special-interest-group" willen ins Blatt gelangen, dann kann sie ihren Leserstamm Schritt für Schritt erweitern. Zielgruppe bleiben aber jene, die sich als wenn auch scheue Christen für religiös-kirchliche Themen interessieren, unabhängig davon, ob sie sich in der Pfarrgemeinde oder einem Verband/Verein ihres Wohnortes engagieren.

Darin läge übrigens eine durch und durch „kat-holische“ Dimension des journalistischen Auftrags: für die eine Gruppe der Leserschaft verständlich und interessant zu machen, wie andere Menschen ihr Leben als Christen zu gestalten suchen und weshalb sie das tun. Die Neugier, das Wissen und die Achtung der einen vor den anderen zu wecken, ist ein lohnendes journalistisches Ziel.

6. Die Redaktion einer Bistumszeitung muß ihre mit dem jeweiligen Herausgeber abgesprochene Linie souverän vertreten. Sie darf sich nicht von außen unter Druck setzen lassen. Die vertretene journalistische Linie muß mit dem Herausgeber abgesprochen sein. $\mathrm{Ob}$ die Herausgeberschaft in allen Fällen beim Bischof oder Bistum liegen muß, ist eine Frage, die von Fall zu Fall und je nach künftige Entwicklung unterschiedlich beantwortet werden kann und muß.

7. Die Redaktion muß mehr Möglichkeiten finden, ihre Leserschaft in einen Dialog zu binden. Von dem, was sich die Kolleginnen und Kollegen anderer Zeitungen zum Thema Leser-Blatt-Bindung bereits haben einfallen lassen, kann sich die Belegschaft einer Bistumszeitung manche Scheibe abschneiden. Allerdings ist dabei eine gewisse Behutsamkeit geboten. Religiöse Menschen sind, so scheint es mir, sehr empfindlich, wenn etwas nur entfernt nach Marktschreierei, Werbung und Gewinn aussieht. Zudem reagiert der moderne Katholik allergisch, wenn er sich vereinnahmt wähnt.

8. Eine vorläufig letzte Herausforderung an die Redaktionen von Bistumszeitungen, gerade in Deutschland, scheint mir darin zu liegen, ihre Blätter unterhaltsamer zu machen. Vor allem hierzulande ist mitunter eine gewisse Verkrampftheit und Verbiestertheit festzustellen, die auch in den Kirchenzeitungen ihren Reflex findet. Geradezu fatal wäre es aber, wenn die Redaktionen der in jüngster Zeit gehäuft auftretenden kirchlichen Paranoia verfielen. Die Zeiten mögen gelegentlich etwas rauher geworden sein für engagierte und bekennende Christen. Weinerliche Defensive aber ist keine Haltung für Blattmacher. Wenn es so weit kommt, daß äußere Feinde herhalten oder gar geschaffen werden müssen, um Leser-BlattBindungen herzustellen - na dann: „Gute Nacht”.

Diese acht Überlegungen widmen sich dem, was von seiten der Redaktion besser gemacht werden kann und muß. Ob es ausreicht, der Bistumspresse bessere Chancen für die Zukunft einzuräumen, oder ob 
die zuvor erwähnten strukturellen Hindernisse unüberwindbar sind, kann ich nicht abschätzen. Entscheiden wird sich das aber im Laufe der nächsten fünf Jahre. Denn dann wird sich herausstellen, welchen weiteren Weg die Bistumszeitungen im einzelnen gehen müssen oder können: Kooperationen, Einstellung, Relaunch, neue Blätter oder weiter so. Auf dem Weg dahin aber ist der intensive Austausch zwischen Redaktion, Verlag und Herausgeber bitter nötig. Wenn überhaupt, dann hat nur eine gemeinsam abgesprochene Strategie Aussicht auf Erfolg.

\title{
Näher an die Menschen heran
}

\author{
von Gernot Facius
}

Goldgelbe Getreidefelder, wogende Ähren, blauer Himmer so weit das Auge blickt. Das Titelbild meiner Kirchenzeitung spiegelt Schönheit, Gelassenheit, Harmonie. Fürwahr: ein Bild des Friedens. In dieser Idylle haben Gedanken an Zwietracht, Konflikt, aber auch an kritischen Diskurs keinen Platz. Eine Momentaufnahme nur. Aber eine symbolkräftige: für die Sehnsucht vieler Kirchenzeitungsmacher nach der heilen Welt.

Scharfzüngige nennen das Gartenlaube-Mentalität. Das mag ja ungerecht sein. Doch wird sich kaum leugnen lassen, daß solche Titelfotos oft auch etwas von der inneren Wunschvorstellung der Herausgeber und Redakteure nach außen kehren: Kirche als unantastbarer Raum der Harmonie; Kirche als blühende Landschaft des Glaubens; Kirche als uneinnehmbare Burg des Heils.

Zugegeben: eine schöne Vision. Aber hält sie der Wirklichkeit stand? Noch konkreter: Wird dieses Bild der Realität gerecht? Kirche ist Gottseidank kein monolithischer Block. Unter dem Dach der Kirche leben die unterschiedlichsten Temperamente, sollten alle Generationen ihren Platz haben - auch die Fragenden, Zweifelnden, "Abständigen" Heimat finden. Wer sich durch das Angebot der konfessionellen Presse wühlt, wird aber das Gefühl nicht los: Hier sollen aus 100prozentigen Katholiken 150prozentige gemacht werden. Herstellen von Öffentlichkeit ja, aber zugunsten der Hierarchie. Unterwürfigkeit wird mit Glaubensgehorsam verwechselt. Im Klima der Ängstlichkeit ersticken hoffnungsvolle Ansätze zu frischer, professioneller Berichterstattung

Gernot Facius ist Ressortleiter bei der Tageszeitung "Die Welt". 
und Kommentierung. Dann bleiben eben als Abonnenten und Leser letztlich diejenigen übrig, deren Kirchenbild noch das alte ist: streng von oben nach unten hierarchisiert, mit möglichst wenig Kritik an den Amtsträgern.

Auflagen und Reichweiten kirchlicher Zeitungen und Zeitschriften gehen kontinuierlich zurück. Die relativ hohe Zahl der Abbestellungen durch Leser unter 40 Jahren verlange die Frage nach der grundsätzlichen Ausrichtung der konfessionellen Presse, sagte im Mai 1996 der Vorsitzende des Fachverbandes konfessionelle Presse im Verband Deutscher Zeitschriftenverleger (VDZ), Hermann Glandorf. Er lieferte eine - einleuchtende - Analyse des Wandels im Leser- und Bezieherverhalten ab: Die Abbesteller von Kirchenzeitungen legten weniger Wert darauf, daß Bistumsblätter die Haltung von Papst und Bischöfen vertreten. Vielmehr erwarteten sie eine ausgewogene Berichterstattung wie in anderen Druckmedien auch. Es müßten auch Meinungen zugelassen werden, die von der offiziellen Kirchenmeinung abwichen.

Das dem König Kunde, sprich dem zahlenden Leser, zu garantieren, ist freilich leichter gesagt als getan. Wenn es, wie geschehen, möglich ist, einem Chefredakteur einer Kirchenzeitung den Stuhl vor die Tür zu stellen, weil er es wagte, in Leserbriefen das Problem der wiederverheirateten Geschiedenen zu thematisieren, wird man kaum erwarten können, daß sich schnell ein Mentalitätswechsel einstellt, der sich auch auf die Auflagenentwicklung positiv auswirkt. Mancher bischöfliche bzw. erzbischöfliche Herausgeber betrachtet "sein" Blatt als willkommenes Instrument zur Fortsetzung seiner Bistumspolitik mit anderen, eben publizistischen Mitteln.

Die Bistumszeitung als verlängerte Kanzel! Was für eine Vorstellung von der Eigengesetzlichkeit der Kommunikationsmittel! In der Konzilszeit sind die Medien einmal "Geschenk Gottes" genannt worden. Heute, in der nachkonziliären Ära, tut mancher Hierarch der Kirche so, als handele es sicher eher um Teufelszeug.

1988 hat sich der Pariser Erzbischof, Kardinal Jean-Marie Lustiger, zur geistlichen Dimension Europas im Hinblick auf die "Neuevangelisierung" geäußert: "Wir müssen in unserer Verkündigung die Freiheit der Kinder Gottes neu entdecken!" Was Lustiger sagt, gilt natürlich auch für den Umgang der Kirche mit den Medien. Groteskerweise hat sich nach dem Fall der Berliner Mauer und nach dem Zusammenbruch des kommunistischen Herrschaftssystems die schon in den achtziger Jahren zu beobachtende ungute Tendenz fortgesetzt, das Freiheitsstreben im Volke Gottes kirchenamtlich "in den Griff" zu bekommen. Es ist schon etwas dran an Hermann Boventers These: „Glaube und Kirche stehen heute zu wenig unter der Autorität des freien Wortes und der Freiheit. Communio kommt von Communicatio. Wo letztere unterbunden oder zu stark reglementiert wird, kommt es nicht zur Communio, 
zur Gemeinschaft im Geist und Heiligen Geist. Es kommt nicht zu einem authentischen und glaubwürdigen Sprechen der Kirche in die Welt hinein. Die Kirche ist zwar nicht von dieser Welt, aber wir leben in ihr, und das geht nicht mit hochgezogener Zugbrücke“ („BekanntMachung", 40 Jahre GKP, 1988).

Das impliziert den Wunsch nach mehr Öffentlichkeit, mehr Raum für das freie Wort. Für Journalisten heißt das: mehr Bereitschaft zur Recherche. „Hinschauen, nicht wegschauen“, formulierte einst - wenn auch in anderem Zusammenhang - Heinrich Böll. Auf die Presse, auch die kirchliche, übertragen, ist das Dichterwort eine Aufforderung zum Hinter-die-bloße-Nachricht-schauen. Das erfordert eine Professionalisierung der publizistischen Berufe. E. Bieger hat in seiner Umfrage „Die Redakteure der Bistumspresse" schon 1978 herausgefunden, daß diese Personen zu 100 Prozent ein überzeugtes Professionsbewußtsein haben: Kirchenblattredakteure müßte "ausgesprochene Journalisten" sein und nicht etwa Theologen oder Seelsorger; die berufliche Arbeit wird "nicht als Form pastoralen Wirkens gesehen, sondern als eigenständige Aufgabe, die eine Professionalisierung voraussetzt. Michael Schmolke wies allerdings ebenfalls schon 1978 (in „Publizistik aus Profession Festschrift für Johannes Binkowski “) darauf hin, daß die Unabhängigkeitszufriedenheit bei Bistumsblattredakteuren niedriger sei als bei Kollegen der Tageszeitungen: 44 Prozent der Bistumsblattredakteure (aber nur acht Prozent der Tageszeitungsredakteure 1973) meinten 1975, daß es manchmal nicht möglich sei, Beiträge zu bringen, auch wenn man sie für wichtig halte, und zwar - so 35 Prozent der Frustierten weil über verschiedene Fragen ,in der Kirchenzeitung nicht offen geschrieben werden" könne. Das liege nicht nur oder immer am Herausgeber, sondern auch an sturen Lesern. Hat sich seither so viel verändert?

Es mag ja sein, daß der Absturz der Kirchenzeitungsauflagen primär mit einer "generellen Glaubenskrise" (Bischof Hermann Josef Spital) zu tun hat. Die Lockerung kirchlicher Bindung hat einen Verlust an religiöser Substanz zur Folge, der sich ohne Zweifel auch in der Bereitschaft, ein kirchliches Medium in die Hand zu nehmen, niederschlägt. Zwischen 1980 und 1994 sank, wie Renate Köcher vom Institut für Demoskopie Allensbach nachweist, der Anteil der katholischen regelmäßigen Gottesdienstbesucher von 38 auf 28 Prozent, und parallel ging der Anteil der Konpress-Leser zurück. Genauso sank die Auflage der Bistumspresse um 30 Prozent. "Überdurchschnittlich ist das Interesse in der Gruppe der Unter-30jährigen und der 30-44jährigen zurückgegangen" (Köcher). Kirchliche Bindungen und Interesse an religiösen Themen hängen eng zusammen. Aber erklärt das schon alles? Vorsicht mit eindimensionalen, man ist versucht zu sagen: einäugigen Deutungen! Der Auflagenverlust und die zunehmend zu beobachtende Konzentration auf die "Kernleser", also die kirchlich fest gebundenen als die primäre Zielgruppe, ging einher mit einer thematischen Verengung. Was sich in der Frühzeit der Republik bei Parteizeitungen 
zeigte und sich später in der (Rück-)Entwicklung bei der Gewerkschaftspresse fortsetzte, schlägt auch hier durch: Innere Haltung, klares weltanschauliches Bekenntnis und pointierte Meinung sind zwar lobenswerte Eigenschaften, aber keine Garanten publizistischen und wirtschaftlichen Erfolgs.

Renate Köcher beschreibt das Problem richtig: "Alle Presseorgane jenseits der aktuellen Tagespresse müssen die Frage nach dem ,Zusatznutzen' beantworten, den sie über die täglich genutzten Medien Tagespresse, Fernsehen und Hörfunk hinaus bieten. Ergebnisse der Inhalts-Analyse der Bistumspresse lassen den Schluß $\mathbf{z u}$, daß viele Bistumsblätter die Frage des Zusatznutzens für sich nicht ausreichend reflektiert haben." Bloßes publizistisches Nachkauen der Ereignisse von gestern, nur auf einer frömmeren Ebene, ist noch kein "Zusatznutzen".

Was könnte aber darunter zu verstehen sein? In erster Linie wohl Orientierungshilfe, „Lebensbewältigungshilfe", Antworten auf die Frage, wie man Kindern den Glauben nahebringen kann. Das setzt allerdings auch voraus, daß man sich über das Bild von Kirche (und vom Menschen) Gedanken macht. Die kirchliche Verkündigung gehe immer vom Bild eines Menschen aus, der an die Zügel genommen werden müsse, beklagt Eugen Biser. „Doch dieser Mensch ist längst einem ganz anderen gewichen, der, weil er unter einem Selbstzerwürfnis leidet und von einer schier unstillbaren Lebensangst gepeinigt wird, in erster Linie der Heilung bedarf." Das Christentum nicht als asketische, sondern als therapeutische Religion - das ruft geradezu nach Konsequenzen für die kirchlichen Medien.

Bistumszeitungen mit ihren bischöflichen Herausgebern legen es darauf an, die Bistumsebene zum Maßstab journalistischer Betrachtungsweise zu machen. Das Wochenblatt als Sprachrohr der Diözesanleitung, als "Hausblatt”, als Spiegel diözesanen Geschehens. „Hausblatt“ muß ja nichts schlechtes sein; im guten Sinne kann darin eine besondere, regionale Handschrift zu erkennen sein. Der Haken ist vermutlich ein anderer. Bischof Spital kommt in einem "Focus"Interview (1995) darauf zu sprechen, als er eine verstärkte Zusammenarbeit der verschiedenen Kirchenzeitungen empfahl: Ein Konzept könnte heißen, die Kirchenpresse müsse zum einen mehr interessante kirchliche Nachrichten aus aller Welt bringen und zum anderen durch Regionalisierung versuchen, wieder näher an die Menschen heranzukommen. Zwar sprach es Spital nicht offen aus, aber es scheint eine Tatsache zu sein: Schon im Wort "Bistumspresse“ zeigt sich eine schädliche thematische, wie Politologen und Soziologen sagen würden, „Engführung“. Die Bistumsebene ist für die Leser so abstrakt (und gedanklich weit weg) wie die Landes- oder die Europapolitik; beide Politikfelder werden in den "normalen" Tageszeitungen, wenn nicht gerade ein Skandal ins Haus steht, auf den inneren bzw. hinteren Seiten beackert. "Die Perspektive der Bevölkerung", heißt es folgerichtig in der Köcher-Studie, ",konzentriert sich auf den Nahbereich und allgemein auf religiöse Themen. Der Nahbereich ist keine Bezugsgröße. ... Die 
Bezugsebenen sind die Großinstitutionen Kirche einerseits und die Gemeinde andererseits."

Erkennt man das als richtig an, ergeben sich daraus logische Folgerungen. Die Kirchenzeitung muß dann stärker ins Lokale bzw. Regionale investierten. Die säkularen Redaktionen haben das schon vorexerziert: All business is local! Das erfordert, auf die kirchliche Presse übertragen, Abkehr vom Terminjournalismus, vom "Abfeiern" von Jubilaren und neuen Trägern päpstlicher Orden und Hinwendung zu einem investigativen Journalismus, natürlich ohne Scheckbuch. Das erfordert aber auch einen neuen Typ von Kirchenblattredakteur. Gesucht ist der Journalist, der ganz für seinen Nahbereich da ist. Der eigenständig recherchiert und reportiert. Der sich durch menschliche Schreibe auszeichnet, der das Diktum verinnerlicht: Information ist nur das, was man versteht! Der also Kirchendeutsch für Otto Normalleser übersetzt. $\mathrm{Ob}$ die Verlage in der gegenwärtigen wirtschaftlichen Flaute bereit sein werden, solche Ideen überhaupt aufzugreifen, muß allerdings bezweifelt werden.

Das ist die Crux der "Kirchenpresse“: Man hinkt hinter den Ergebnissen der Medien- und Leserschaftsforschung hinterher. Betrüblich. Wer für ein säkulares Medium tätig ist, bekommt täglich zu spüren, wie groß das Interesse des Publikums an religiösen und kirchlichen Fragen ist - auch der zu den „Distanzierten“ gerechneten Leser. Das scheint auch die Untersuchung des „psychologischen Interessenpotentials" zu bestätigen. Dieses noch nicht ausgeschöpfte Leser- und Abonnentenpotential findet sich nicht nur unter kirchennahen Katholiken, die fast jeden Sonntag zum Gottesdienst gehen (48 Prozent), sondern auch bei kirchenfernen Katholiken (22 Prozent), nicht nur bei Kirchentreuen (47 Prozent), sondern auch bei Katholiken, die von sich sagen: „ich fühle mich als Christ, aber die Kirche bedeutet mir nicht viel“" (26 Prozent).

"Kirche", was immer man auch im einzelnen darunter verstehen mag, ist in Tages- und Wochenzeitungen längst nicht mehr in ein Ghetto verbannt. "Kirche" hat bereits die Titelseiten erobert: manchmal zu stark personalisiert, an Konflikten orientiert, das "Exotische" nach außen kehrend. Aber auch hier setzt sich das Prinzip der Branche durch: "Nachricht ist, was sich verändert." Dieser medialen Eigengesetzlichkeit und Dynamik kann die "normale" Tageszeitung leichter folgen als das Bistumsblatt. Aber letzteres ist in der Lage, hinter die Nachricht zu schauen, sie einzuordnen, korrigierend zu wirken, wo die Verzerrung zu offensichtlich ist. Zwischen beiden Medienebenen könnte sich ein fruchtbares Spannungsverhältnis entwickeln, wenn jede sich auf ihre spezifische Stärke besinnt. Vielleicht ist das ja zu idealistisch gedacht. Aber man sollte es immer probieren. Über eins sollte man sich allerdings auch im klaren sein: Mit einem schönen Layout allein wird man den Bezieherschwund nicht stoppen. Verbesserte Optik plus mehr Sorgfalt, den Leserinteressen nachzuspüren - damit könnte man es schaffen. 


\title{
Ein entschiedener Wille tut den Kirchenzeitungen not
}

\author{
von Peter Schilder
}

Zahlen sind gut. Zahlen sind besonders gut für Menschen, die Zahlen mögen, die ihnen vertrauen. Zahlen können Argumente stützten, können gar selbst zu Argumenten werden. Zahlen kann man wenden, bündeln, zusammenstellen. Man kann damit jonglieren, wenn man jonglieren kann, und man kann damit um sich werfen, wenn man sich auf die Kunst des Jonglierens nicht versteht. Zahlen taugen zu vielem, und das ist ihre Crux. Das scheint auch die Crux der Demoskopie zu sein. Nur selten verschaffen Zahlen und Fragen und Antworten neue Einsichten, bestenfalls bestätigen sie eigene Erfahrungen, machen richtige Vermutungen zur dokumentierten Gewißheit. Bestimmt aber ersetzen Zahlen und demoskopische Fragen nicht Entscheidungen, wirtschaftliche und politische Absichten. Der Wille, die Absicht, die Entschlossenheit gehen jeder Erhebung voraus. Wer weiß, was er (oder sie) will, wird Statistik und Demoskopie zu nutzen wissen. Natürlich in seinem Sinne.

Derlei Vorbemerkungen treffen auch auf die Allensbach-Erhebung zur Bistumspresse zu. Sicherlich hat die Erhebung eine ganze Menge Arbeit gemacht. Sie hat auch entsprechend viel Geld gekostet, Kirchensteuermittel. Dabei herausgekommen sind manche tiefgreifenden Erkenntnisse: z.B. daß Bischöfe und Chefredakteure von Kirchenzeitungen nahezu gleichermaßen der Auffassung sind, daß Kirchenzeitungen sowohl die "Haltung von Papst und Bischöfen entscheidener vertreten" als auch "für kritische Meinung offen" sein sollten. Ungefähr 60 Prozent sind dieser Meinung. 57 Prozent umfaßt die größte Lesergruppe. $\mathrm{Zu}$ ịhr gehören Personen, die älter als 60 Jahre alt sind. So, so, mag mancheiner dabei denken, und sich hinter dem Ohr kratzen. In Zahlen hat er es bisher nicht ausdrücken können, aber neu ist das auch nicht. Zumindest geahnt, wenn schon nicht gewußt, hat das doch fast jeder, der sich mit diesen Fragen befaßt. Es stände ziemlich schlimm um die katholischen Verlage, wenn vieles davon nicht aus ihren Akten hervorginge. Aber wenn eine neutrale Instanz zu denselben Einsichten kommt, wie man selbst, ist das auf alle Fälle wohltuend.

Nein, das soll hier keine Satire werden. Das könnten andere wirklich besser. Aber nunmal ehrlich, was hat denn die AllensbachUntersuchung wirklich Neues gebracht? In welcher Weise trägt sie dazu bei, neue Entscheidungen zu treffen, die der Bistumspresse eine neue

Peter Schilder ist Redakteur der „Frankfurter Allgemeinen Zeitung". 
Chance gäben? Ist nicht genug erhoben worden? Wissen nicht längst alle, die es angeht, daß es so nicht weitergeht? Versucht man sich nicht durch nochmaliges und nochmaliges Untersuchen vor der dringend notwendigen Entscheidung zu drüicken? Mangelt es nicht einfach an Mut, der Ahnung, die oft längst Überzeugung ist, zu folgen. Wäre das nicht das angemessene unternehmerische Verhalten oder - kirchlich gewendet - das Handeln im Vertrauen auf Gottes wohlwollenden Segen? Was ist eigentlich los mit der Bistumspresse?

Es ist bekannt, daß fast alle Kirchenzeitungen mit dem Rücken zur Wand stehen. Da reißen sich ganze Redaktionen, die nicht schlechter sind als andere, Beine und Haare aus, und dennoch übertrifft die Zahl der Abbestellungen - meist nach Todesfall - die Zahl der Neubestellungen ganz deutlich. Der Trend auf dem Zeitungsmarkt ist eigentlich ganz gegenläufig. $\mathrm{Da}$ "sterben" zwar auch Titel, oft sind diese gar nicht alt geworden, aber es werden auch dauernd neue Zeitschriften und Magazine auf den Markt gebracht, die sich dann behaupten und ihren Verlegern reichlichen Ertrag einbringen. Das gilt besonders auf dem Sektor der Sparten- und Spezialzeitschriften, zu denen ja - auch nach Allensbach - die Kirchenzeitungen zu zählen sind. So schlecht muß es also gar nicht um die Bistumszeitungen stehen.

Allerdings wären da wieder einige Vorklärungen nötig, besser gesagt vorausgehende Entscheidungen. Sollen sich die Bistumszeitungen überhaupt auf dem freien Markt behaupten oder sollen sie Mitgliederzeitschriften oder Mitteilungsblätter sein? Auch auf die Frage gibt es wie immer wenigstens zwei Antworten. Über jede wird man reden können und müssen, aber Klarheit muß sein. Wenn die Entscheidung für die sogenannte marktwirtschaftliche Lösung, die angesichts der knappen Kassen nahe liegt, gefallen ist, dann muß man sich den Bedingungen des Marktes stellen. Das heißt noch lange nicht, daß man alle Bedingungen akzeptiert, aber man muß sich damit auseinandersetzen. Wer von seinen Lesern gekauft werden will, wer also zu jedem Erscheinungstag den Kampf zwischen Konsum und Abstinenz gewinnen will, muß seinen Lesern etwas bieten. Vorsicht, das bedeutet nicht nur leichte Kost und ist auch nicht mit einem Hinweis darauf wegzuwischen. Die Leser sind anspruchsvoller als viele glauben, und sie merken, wenn sie verkohlt werden. Nun wäre im einzelnen zu fragen, was das bedeutet, was der Leser will, und was die Redaktion anzubieten hat. Die Antworten darauf würden diesen Rahmen hier sprengen. Aber diesen Fragen nicht nur demoskopisch, sondern auch ehrlich - was wirklich nicht ausschließend gemeint ist - nachzugehen, würde zu vielen zum Teil verblüffenden Einsichten führen, über die am Ende allerdings wieder entschieden werden müßte.

Es gibt auch die andere Möglichkeit, nämlich die Kirchenzeitungen als Mitteilungsblätter zu führen. Nur sollte niemand dabei dem Trugschluß erliegen, daß der Leser dabei weniger wichtig sei. Das Blatt soll doch gelesen werden. Bei der Fülle von Gedrucktem, die jedem 
heute ins Haus flattert, will der Mensch schon etwas davon haben, sonst gibt es schönere und sinnvollere Beschäftigungsmöglichkeiten. Auch ein Mitteilungsblatt muß seine Leser gewinnen. Also ist auch das keine Einbahnstraße. Dafür gibt es vieles zu bedenken und zu berücksichtigen. Das Wissen davon ist in den Redaktionen und Verlagen sowie in unmittelbaren und mittelbaren Kirchenkreisen durchaus vorhanden. Man müßte nur Gebrauch davon machen.

Die ganze Debatte um die Kirchenzeitungen - auch das hat Allensbach aufwendig festgestellt - weist auf eine viel schwierigere Frage hin: mit der Kommunikation nach innen und außen hat die katholische Kirche offenbar ein Problem. Das ist nicht so leicht zu beheben. Das hat seine Ursache in der Struktur der Kirche, die nun einmal hierarchisch organisiert ist und lieber vermeldet als bespricht. Aufmerksamkeit verdient in diesem Zusammenhang auch die Kommunikationsinitiative von vier Bistümern mit einer PR-Agentur, die in diesen Tagen beendet wurde. Das ist zwar vordergründig ein anderes Thema, aber vielfältige Konvergenzen scheinen dabei auf. In dieser Hinsicht ist zwar schon einiges in Bewegung geraten, aber es ist nicht zu erwarten, daß sich das Kommunikationsverhalten bald und grundsätzlich ändert. Deshalb ist auch nicht zu erwarten, daß das "Problem" der Bistumszeitungen bald gelöst wird und ihre Chancen genutzt werden. Da besteht ganz offensichtlich ein Zusammenhang.

Aber man könnte ja mit den Kirchenzeitungen anfangen und müßte nicht die Grundsatzfrage auf den Tisch bringen, auch wenn sie sich nicht raushalten läßt. Aber auch das setzt eine Entscheidung voraus; man müßte es wollen. Wenn man es dann will, dann würden auch die Mittel bereit gestellt werden; denn auch die Chancen sind nicht umsonst zu nutzen. Große Verlage wenden Millionenbeträge dafür auf - und setzen sie gelegentlich in den Sand. Das muß die Kirche nicht unbedingt nachmachen, aber sie muß endlich wissen, was sie will, und was ihr die eigenen Medien wert sind. Solange darüber keine Entscheidung gefallen ist, bleiben die Bistumszeitungen mit dem Rücken an der Wand und verlieren täglich Terrain, auf dem andere bauen. Das ist zwar noch weit von einem Patentrezept entfernt, aber es ist die Voraussetzung, damit das jeweils richtige Rezept gefunden werden kann. 


\title{
Die dahinsiechende Bistumspresse
}

Ginge es nicht doch anders?

\author{
von David Seeber
}

Dies Bistumspresse kränkelt dahin. Je mehr Pressemediziner und demoskopische Heilpraktiker sich um sie bemühen, um so offensichtlicher wird das Siechtum. Jede neue Expertise bringt mehr oder weniger denselben Tatbestand zutage. So kann es nicht weitergehen.

Man will dennoch nicht aufgeben, hofft durch Zielgruppenstrategien und Personalentwicklungspläne zu guter Letzt doch noch das nötige Publikum zu erreichen und klammert sich dabei an irgendwelche Wunschvorstellungen von Sprache, Aufmachung, Inhalt und Problembewußtsein der Redaktionen, Verleger und Herausgeber. Selbst die Quadratur des Kreises, die Zusammenbindung von Alt und Jung und deren auseinanderstrebende Kulturen und Lebensstile, traut man sich mehr aus Willen denn aus Einsicht zu.

Aber was für manche Diözese einst ein willkommenes Zubrot war, ist inzwischen längst zu einem lästigen, aber anscheinend unvermeidlichen Subventionsobjekt geworden. Die seit Anfang der sechziger Jahre praktisch halbierten Abonnentenzahlen sinken überall weiter, wenn auch nicht in jeder Diözese im selben Tempo. Die Überalterung der Leserschaft schreitet fort. Irgendwann wird es auch dem willigsten Bischof des Zuschießens zuviel werden. Der fatale Ausgang ist folglich abzusehen, wenn nicht für heute, so doch für morgen oder spätestens übermorgen. Alle „Stilbücher", Resonanzanalysen und Trainingslager für Redakteure werden daran nichts ändern.

Unermüdlich werden dennoch Varianten überlegt, wie man durch Konzentration der Kräfte doch noch zum Erfolg kommen könnte, z.B. durch verstärkte regionale Kooperation, die mit dem - verständlichen Eigenleistungswillen der Redakteure nicht so recht in Einklang zu bringen ist, oder durch einen gemeinsamen Mantel als gesamtdeutsche Hülle für einen informativ ausgestalteten und regionalen Bedürfnissen Rechnung tragenden Diözesananteil, was wiederum manchem bischöflichen Herausgeber nicht ins Konzept paßt.

Aufschlußreich ist, daß man die Ursachen des sich beschleunigenden Mißerfolges neben dem Säkularitätsdruck nach wie vor hauptsächlich in mangelnder Professionalität zu finden glaubt, als ob das Produkt

David Seeber war von 1966-1991 Chefredakteur der Herder-Korrespondenz und von 1991-1996 Grundsatzreferent im Staatsministerium in Stuttgart. 
schlechter wäre als die Umstände es erlauben und als ob es nur am guten Willen läge, Spitzenkräfte für eine Bistumszeitung zu gewinnen, die ohne große Gehaltsansprüche ihre Arbeit mit optimalem Können und letztem Einsatz tun.

Seltsamerweise wird noch kaum ein Gedanke darauf verwendet, ob die "Printgattung" Bistumspresse nicht in sich überholt ist, sofern sie überhaupt je ein nachahmenswertes Modell war.

Indessen kann bereits ein kurzer Blick ins benachbarte Ausland zeigen, wie berechtigt ein solches Nachdenken wäre.

Es zeigt sich nämlich dabei als erstes, daß Bistumszeitungen als Kernbestand kirchlicher Publizistik in der hierzulande üblichen Aufmachung und dem damit verbundenen Anspruch eine bundesdeutsche Spezialität sind (sieht man von gewissen, auf ganz anderen geschichtlichen und aktuellen Voraussetzungen fußenden Parallelen in den USA ab), typisch nicht einmal für den gesamten deutschen Sprachraum (für die Schweiz nicht, in Österreich zogen kleinere Diözesen in Aufmachung und Inhalt erst seit den sechziger Jahren nach).

Und - sehr viel wichtiger - als zweites fällt auf, daß Länder ohne eine nennenswerte Bistumspressetradition über eine vielgestaltigere katholische Presse verfügen (und teilweise auch bei der Handhabung der neuen lokalen und regionalen Möglichkeiten in den elektronischen Möglichkeiten agiler sind), als dies in Deutschland der Fall ist.

Die französischen Katholiken haben ihre "la croix", die sich trotz konfessioneller Prägung und finanziellen Engpässen mit einem immer noch leistungsfähigen Verlagshaus im Hintergrund mit den besten französischen Tageszeitungen messen kann. Im Gegensatz zu Deutschland verfügt der französische Katholizismus auch noch über eine beachtliche regionale Presse. Und ein Produkt wie den "Pelerin“ sucht man in Deutschland vergeblich.

Italien, kirchlich wie politisch übermäßig gezeichnet von der Nähe Roms, hat heute zwar keine überlebenssichere katholische Tageszeitung mehr, die mit säkularen Medien konkurrieren könnte, dafür leistet sich der italienische Katholizismus, vielfach von Orden getragen, ein sehr viel vielfältigeres katholisches Zeitschriftenwesen als es Deutschland aufzuweisen hat - von kirchlichen Fachorganen mit politischer Antenne bis zur großen Publikumszeitschrift. "Famiglia cristiana" mit einer Auflage von immer noch über einer Million ist aufgrund ihrer journalistischen Aufmachung und meinungsfreudigen Kommentierung kirchlicher wie politischer Fragen zu einer nationalen Institution geworden. Und selbst der schmalschultrige britische Katholizismus hat in "Tablet" ein Organ, wie man es in Deutschland zur Zeit vergeblich sucht. 
Selbstverständlich haben auch alle diese größeren und kleineren Produkte gegen nachlassendes Interesse anzukämpfen und bekommen die Probleme der innerkatholischen Pluralisierung zu spüren, aber sie verkörpern noch eine in der Substanz gesunde, wenn auch in Ausmaß und Wirkung begrenzte katholische Publizistik. In Deutschland dagegen läßt sich nach dem bereits mit der Gründungskonzeption vorgegebenen Scheitern von "Publik" von einer katholischen Presse von einiger Konsistenz nicht mehr sprechen - nicht bei den zu sehr an binnenkirchlichen Belangen klebenden Zeitschriften und erst recht nicht bei der Tages- und Wochenpresse.

Die zentrale Stellung der Bistumspresse in Deutschland, begründet ursprünglich im erzwungenen Rückzug auf die binnen- und amtskirchlichen Strukturen während des Dritten Reiches und haltbar nur in einem Land mit übergroßen Diözesen, hat zum einen der inzwischen als drückend empfundenen Verkirchlichung (Veramtlichung) auch im Pressewesen vorgearbeitet und der Erhaltung einer freien katholischen Presse mehr geschadet als genutzt.

Zum anderen fehlt gerade der Bistumspresse zur vollen professionellen Entfaltung schlicht die notwendige Unabhängigkeit. Man muß Forderungen nach Verwirklichung des Forumscharakters von Presseerzeugnissen nicht übertreiben, aber die bischöfliche Herausgeberschaft ist kein guter Nährboden für eine profilierte Publizistik. Ich möchte den Bischof kennen, der, zumal angesichts höchst empfindlich gewordener römischer Beobachter, nicht schon beim ersten Versuch einer pointiert abweichenden Meinung zu einem der großen Moraloder Gesellschaftsthemen in Autoritätsverstrickungen gerät und das den verantwortlichen Redakteur auf dessen Kosten spüren läßt. Da dieser ja Bescheid weiß und seine berufliche Existenz daran hängt, läßt er die heißen Themen gleich sein.

Katholische Presse gedeiht nur in der Hand von kirchlich loyalen, aber nicht beruflich und finanziell von der Hierarchie abhängigen katholischen Laien oder unter der Obhut von Ordensgemeinschaften mit dem nötigen Stehvermögen und einem ausreichenden publizistischen Know-how.

Natürlich bedarf es neben einer katholischen freien Presse kirchenamtlicher oder kirchamtlich verantworteter Kommunikation. Aber warum angesichts der offensichtlichen und nicht behebbaren Schwächen der Bistumspresse nicht auch da - und insgesamt - neue Wege gehen, auch wenn das tempus utile für manches Projekt möglicherweise längst vorbei ist?

Drei Linien halte ich für entwicklungsfähig:

Im kirchenamtlichen Bereich ein anzeigenfinanziertes Mitteilungsblatt mit Diözesanbeilagen, das über die wichtigen, die Kirche und ihr 
gesellschaftliches Umfeld betreffenden Ereignisse in Deutschland, in den einzelnen Diözesen und in der Weltkirche informiert und jedem Katholiken (jedem Haushalt von Katholiken) unentgeltlich zugestellt wird. Angesichts des sehr ausgeprägten deutschen Regionalismus wird so etwas kaum gesamtdeutsch zu machen sein (die Bayern würden wohl auf keinen Fall mitmachen), sondern nur über überregionale Ausgaben (eventuell, wie in anderem Zusammenhang schon öfter ventiliert, mit einem gemeinsamen gesamtdeutschen Mantel). Wenigstens sollten aber gemeinsame redaktionelle Teile und eine Anzeigengemeinschaft möglich sein.

Parallel dazu bräuchte es auf der unteren Ebene (Landkreis, Gesamtkirchengemeinde kreisfreier Städte) lokale kirchliche Mitteilungsblätter. (Die Schweizer machen mit ihren übergemeindlichen „Pfarrblättern“ bis heute gute Erfahrungen; in den Städten und Kreisen spielt sich das erfahrbare bürgerliche Leben ab; das überörtliche "Lokale" wird für katholische Gemeinden und die Katholiken einer Region schon allein wegen des immer größeren Klerusmangels von Jahr zu Jahr wichtiger.)

Die Bischöfe wenden sich noch überwiegend gegen solche "Blatt-Formen". Sie vermuten darin reine Mitteilungsblätter und sehen durch sie überdies ihre diözesane Suprematie verletzt. Aber das erstere ist ein Mißverständnis: Ein anzeigenfinanziertes Mitteilungsblatt muß sich nicht auf Informationen über Personalien und organisatorische Vorgänge und die jährliche Publikation kirchlicher Etats beschränken. Gute informative Sach- und auch Meinungsartikel zu kirchenpolitischen oder gesellschaftlichen Vorgängen lassen sich damit sehr wohl verbinden. Und als kostenlos zugestelltes Blatt, das an alle Haushalte geht, hat es überdies eher die Chance, auch manche Fernstehende zu erreichen, als ein von vornherein auf ein binnenkirchlich interessiertes älteres Publikum zugeschnittenes Bistumsblatt.

Und das Letztere - die bischöfliche Suprematie betreffend? Da werden wohl die heute wirksamen sozialpsychologischen Bindungsmechanismen falsch eingeschätzt. Renate Köcher stellt fest, die Bistumsebene sei keine Beziehungsgröße, genausowenig wie die Landespolitik. Für die Landespolitik -kann dies nur nachdrücklich bestätigt werden, und für die Bistumsebene gilt es erst recht - gerade wegen der übergroßen Diözesen und deren dem geschichtlichen Zufall zu verdankenden Grenzen.

Eine zweite Entwicklungslinie: Wenn die Bistumspresse ohnehin schon Kirchenpresse weitestgehend der älteren Generation ist, warum sich dann nicht gleich und entschieden für eine generationenspezifische und in erster Linie an lebensweltlichen Identitäten orientierte Spezialpresse stark machen: für eine nicht penetrant das Alter herausstellende Seniorenpresse z.B., solange dieses Terrain noch nicht nach allen Seiten besetzt ist? Paralleles gilt - Versuche werden von der Weltbild-Gruppe ja gemacht - für die Familien- und Frauenpresse und trotz aller 
Schwierigkeiten auch für ein locker gestaltetes katholisches Jugendmagazin.

Und - dritte Linie - da die Zeit für eine große katholische Wochenzeitung und für katholische Tageszeitungen, die über kirchliche Rest- und Sondermilieus hinausreichen, erst recht längst vorbei ist, müßten sich die Katholiken in Deutschland zumindest ein Multiplikatorenorgan leisten können - in Gestalt etwa eines anspruchsvollen Monatsmagazins von einem intellektuellen Niveau, an dem niemand ganz vorbeigehen kann, der sich mit religiös-ethischen, mit gesellschaftspolitischen Fragen und mit wissenschaftlich-technischen Errungenschaften, soweit diese das Selbstverständnis des Menschen berühren und sein Gewissen herausfordern, auseinandersetzen will. Es gibt noch einige wenige gute Informations- und Kulturzeitschriften im katholischen Bereich, aber keine von ihnen erfüllt gegenwärtig diese Funktion.

Natürlich steht und fällt das alles mit den verlegerischen Persönlichkeiten, die sich einer solchen Sache annehmen, und Verlegerpersönlichkeiten lassen sich, zumal in einem von Verzagtheit und Selbstzweifeln umgetriebenen Katholizismus, nicht aus dem Boden stampfen. Aber zumindest sollte man wissen, was man will. Die demoskopische Erfassung von Meinungssegmenten, die ohnehin nie ein operables Ganzes ergeben, ist dafür kein Ersatz.

\title{
Keine Orientierung an der Kirchenpresse Zur Stellung der Kirchen innerhalb des Mediensystems
}

\author{
von Michael Hertl
}

Die Kirchenpresse sei heute, so stellen Hans Mathias Kepplinger und Simone Ehmig in der Einleitung der Ergebnispräsentation ihrer Inhaltsanalyse der Bistumszeitungen fest, „für die Öffentlichkeit jenseits der Kern-Zielgruppe bis zur Bedeutungslosigkeit geschrumpft ${ }^{\prime} .{ }^{1}$ Wichtiger noch als der Verlust des Kontaktes zu weiten Teilen der Bevölkerung sei aber, daß damit auch die eigenständige Präsenz der Kirchen innerhalb des Mediensystems verlorengegangen sei. „An ihren Publikationen muß sich - von Ausnahmen abgesehen - ein Journalist nicht mehr orientieren. ${ }^{2}$

Michael Hertl ist freier Mitarbeiter der Kirchenzeitung "Der Sonntag" in Limburg.

1 H. M. Kepplinger/S. Chr. Ehmig, Form und Inhalt der Bistumspresse. In: Communicatio Socialis 29 (1996) 3. 
Ist also der Dialog zwischen "Kirche und Welt" damit beendet? Muß der Versuch, Kirche und Massenmedien miteinander zu versöhnen, der im Anschluß an "Communio et Progressio“ unternommen wurde, als gescheitert angesehen werden? Erfüllt die Kirche also überhaupt noch ihren Auftrag, zur Bildung von öffentlicher Meinung beizutragen, auch in dem immer größer werdenden, nicht bereits stark kirchenorientierten Gesellschaftssegment? Oder ist die Wirkung der Kirchenpresse tatsächlich nur noch auf den binnenkirchlichen Raum beschränkt, wo sie als eine Art „Vereinsblatt“ fungiert?

\section{Ein Fallbeispiel}

In wesentlichen Teilen werden die diesbezüglichen Ergebnisse der Studie von Kepplinger/Ehmig in einer Untersuchung bestätigt, die sich mit der Berichterstattung über den Konflikt zwischen Eugen Drewermann und der Katholischen Kirche in ausgewählten deutschen Wochenzeitungen beschäftigte. ${ }^{3}$

Ebenfalls mittels einer Inhaltsanalyse wurde am Beispiel eines konkreten Ereignisses untersucht, wie sich die Berichterstattung in kirchlich "gebundenen" und kirchenfernen Periodika unterscheidet. Für eine Untersuchung der meinungsbildenden Wirkung kirchlicher Zeitungen war ein Thema zu wählen, bei dem man der Kirche eine bestimmte Kompetenz zuerkennt. Ein solches Thema war die Auseinandersetzung, die zwischen dem Paderborner Theologen Eugen Drewermann und seinem Ordinarius, Erzbischof Johannes Joachim Degenhardt, dem Oberhirten des Erzbistums Paderborn, zwischen Oktober 1989 und Januar 1992 ausgetragen wurde. Kompetenz sollte die Kirche in diesem Fall besitzen, weil es ja zum einen um explizit theologische Differenzen, „Lehrstreitigkeiten“, ging, zum anderen, weil alle vom Konflikt betroffenen Personen aus dem Bereich der Kirche stammten. In weiten Teilen der Massenmedien wurde über diesen Konflikt berichtet, so daß er einer breiten Öffentlichkeit bekannt wurde. Das Bild, das sich die Öffentlichkeit von der Katholischen Kirche machte, wurde zumindest im näheren zeitlichen Umfeld des Konflikts durch die Berichterstattung darüber wesentlich beeinflußt. Weil kirchlicherseits der Eindruck entstand, daß das Bild der Katholischen Kirche von den Medien zu großen Teilen verfälscht dargestellt wurde, beklagte man dort die „publikumswirksame“ Tätigkeit Drewermanns. Nur wenig beachtet wurde hingegen, daß auch die Kirche selbst über ihre eigenen oder ihr nahestehende Publikationsorgane zu den Berichterstattern des Konflikts gehörte und somit die Berichterstattung selbst aktiv beeinflussen konnte. An diesem Fall war also konkret zu überprüfen, ob die Kirche

3 Vgl. M. Hertl, Die Berichterstattung über den Konflikt zwischen Eugen Drewermann und der Katholischen Kirche in ausgewählten deutschen Wochenzeitungen. Unveröffentlichte Magisterarbeit. Mainz 1994. 
ihre publizistischen Möglichkeiten nutzte, um auf die öffentliche Meinung einzuwirken, oder ob sie dazu nicht in der Lage war.

\section{Exkurs: Agenda-Setting}

An dieser Stelle soll kurz noch einmal an das kommunikationswissenschaftliche Konzept des „Agenda-Setting" erinnert werden.

Die Kommunikationswissenschaften haben den Begriff des "Agenda-Setting" eingeführt, um zu beschreiben, in welcher Weise die in Teilen der Massenmedien stattfindende Berichterstattung selbst wieder zur Thematisierung in der Berichterstattung anderer Medien und schließlich zur Meinungsbildung in der Gesellschaft beitragen kann. Denn beim Prozeß der Meinungsbildung spielen Journalisten ja bekanntermaßen eine maßgebliche Rolle: Nach dem Konzept des Zwei-Stufen-Flusses der Kommunikation spielen Journalisten die Rolle von Meinungsführern, die insbesondere bestimmte "Bezugsrahmen " bei der Berichterstattung über ein Thema aufbauen (,framing “) und so die Einordnung eines Themas in einen bestimmten Wahrnehmungshorizont vornehmen. Es kommt dabei innerhalb des Mediensystems zu einer "Ko-Orientierung", einem „inter-media agenda-setting“, bei dem die Medien sich gegenseitig beeinflussen und Kettenreaktionen auslösen. Es müssen dabei aber nicht immer die "Meinungsführermedien" sein, die die Rolle des Meinungsführers spielen: auch "alternative“ Medien oder solche, die eine besondere Kompetenz besitzen, können unter Umständen diese Rolle spielen. 4 Unter Umständen könnten also durchaus auch kirchliche Medien die Rolle eines solchen „Meinungsführermediums" übernehmen.

\section{Auswahl der Zeitungen}

In erster Linie denkt man bei den "kirchlichen Medien" natürlich an die Kirchen- oder Bistumszeitungen, die ja auch im Mittelpunkt der Untersuchungen von Kepplinger/Ehmig, Schulz und Köcher standen. Bei diesen Zeitungen fungieren die deutschen Bistümer selbst als Herausgeber und bestimmen somit auch, mehr oder weniger direkt, die redaktionelle Linie dieser Blätter. Für die Untersuchung der Drewermann-Berichterstattung wurden "Der Dom" aus Paderborn und "Glaube und Leben" aus Mainz ausgewählt, letztere wurde dabei aus verschiedenen Gründen als repräsentativ für die Kirchenzeitungen der deutschen Bistümer gewertet. 5 Darüber hinaus besitzt die Katholische Kirche in Deutschland aber als Gesellschafter auch einen bestimmenden

4 Vgl. E. Noelle-Neumann u.a. (Hrsg.), Fischer Lexikon Publizistik Massenkommunikation. Frankfurt 1994, 554-571.

5 So wird der Mantelteil von "Glaube und Leben", "Der Sonntag" und „Bonifatiusbote" von einer gemeinsamen Redaktion der Bistümer Fulda, 
Einfluß auf die Wochenzeitung "Rheinischer Merkur" (RM), der deshalb in die Untersuchung einbezogen wurde.

Man kann davon ausgehen, daß in diesen "katholischen“ Zeitungen die Sicht der Kirche relativ unverfälscht wiedergegeben wurde, mit anderen Worten: diese Zeitungen berichteten über den Konflikt so, wie er sich aus Sicht der Katholischen Kirche darstellte. Für die Öffentlichkeit ist dieses Bild jedoch - zumindest unmittelbar - nicht maßgeblich. Die außerhalb der Kirche stehende Öffentlichkeit bildet sich ihre Meinung nicht durch das Lesen kirchlicher Zeitungen, sondern zum Beispiel durch Informationen aus politischen Wochenmagazinen wie dem "Spiegel" oder der "Zeit". Beide Magazine wurden deshalb in die Untersuchung miteinbezogen.

Die einzige Möglichkeit, den kirchenfernen Teil der Öffentlichkeit doch zu erreichen, besteht für die kirchennahen Zeitungen in einer Einflußnahme auf den Rahmen, in dem die Berichterstattung durch die anderen Medien stattfindet. Dies wird als "framing" bezeichnet. Damit ist gemeint, daß man über jeden Sachverhalt aus grundsätzlich verschiedenen Perspektiven berichten kann. Im vorliegenden Fall etwa setzte sich der Konflikt aus einer theologischen, einer systemtheoretischkirchenkritischen und einer konfliktbetonenden Ebene zusammen. Während Drewermann und Degenhardt zuerst anscheinend nur über konkurrierende theologische Ansätze stritten, weitete sich ihr Streit schon bald auf grundlegende Fragen der Ekklesiologie, zu Macht, Amt und Hierarchie in der Kirche aus und nahm schließlich die typischen Züge eines gewöhnlichen Konflikts an. Diese drei Ebenen überlagerten sich und traten im Zeitverlauf unterschiedlich deutlich in den Vordergrund. Maßgeblichen Einfluß auf das öffentliche Bild vom Konflikt in der Öffentlichkeit besaß deshalb derjenige, der Einfluß auf die Akzentuierung dieser Ebenen nahm und damit den Rahmen der Berichterstattung bestimmen konnte. War es ein eigentlich theologischer Streit? Wer hatte darin die besseren Argumente? Oder trat in dieser Auseinandersetzung eine viel grundlegendere Problematik zutage und es ging eigentlich um die Macht in der Institution Kirche? Oder ging es vielleicht gar nicht um inhaltliche Fragen, sondern nur um das aggressive Verhalten eines Einzelnen innerhalb einer Institution? An diesen Fragen wird deutlich, daß in der Definition der Auseinanderset-

Mainz und Limburg herausgegeben. Diese Bistümer können von ihrer demographischen Struktur her als repräsentativ für die (alten) Länder der Bundesrepublik gelten. Hinzu kommt, daß die Bischöfe dieser drei Diözesen sich in ihrem ekklesiologischen Selbstverständnis deutlich unterscheiden und somit in ihrer gemeinsamen Bistumszeitung eine redaktionelle Linie finden müssen, die den verschiedenen Perspektiven gerecht wird. Drittens kann man annehmen, daß gerade die Kirchenzeitung aus dem Bistum Bischof Karl Lehmanns, des Vorsitzenden der Deutschen Bischofskonferenz, um eine vermittelnde, möglichst breite Zustimmung hervorrufende Berichterstattung und Kommentierung bemüht sein wird. 
zung der Schlüssel zur Meinungsführerschaft lag. Um diese Einflußnahme (direkt auf die Leser und indirekt über die Meinungsführermedien) „konkurrierten“ nun im untersuchten Konflikt um Eugen Drewermann kirchliche und kirchenkritische Zeitungen, wie etwa "Publik-Forum“ miteinander. Neben dem "Publik-Forum" wurde schließlich noch das Pendant der evangelischen Kirche zum "Rheinischen Merkur", das "Sonntagsblatt“ (zum Zeitpunkt der Untersuchung noch "Deutsches Allgemeines Sonntagsblatt“, darum im Folgenden abgekürzt als „DAS“) in das Sample aufgenommen.

\section{Modelle der Berichterstattung}

Vergleicht man nun den Inhalt der Berichterstattung der katholischen Presse mit dem in kirchenkritischen und in kirchenfernen Zeitungen, kann man verschiedene Konstellationen bezüglich der Übereinstimmung erwarten. Grundsätzlich können sich drei Modelle ergeben:

1) Kirchenkonforme Übereinstimmung:

Kirchliche und kirchenferne Berichterstattung sind im wesentlichen gleich. Die kirchenferne und die kirchliche Offentlichkeit werden so in gleicher Weise informiert. Setzt man die Existenz medieninterner Orientierungsprozesse voraus, kann man folgern, daß die kirchenferne Presse sich an der Kirchenpresse orientiert, da diese im konkreten Fall "näher am Geschehen" ist und eine themenspezifische Fachkompetenz besitzt. Die kirchenkritische Presse nimmt in dieser Konstellation eine Außenseiterposition ein.

2) Unterschiedliche Berichterstattung:

Die Berichterstattung der kirchenfernen Presse unterscheidet sich deutlich von der der Kirchenpresse und der kirchenkritischen Presse. Kirchliche, kirchenkritische und kirchenferne Öffentlichkeit werden unterschiedlich informiert. Eine medieninterne Orientierung an der Kirchenpresse oder der kirchenkritischen Presse findet nicht statt.

3) Kirchenkritische Übereinstimmung:

Die Berichterstattung von kirchenkritischer und kirchenferner Presse ähnelt sich stark, während die kirchlichen Medien davon verschieden berichten. In diesem Fall ist eine Orientierung der kirchenfernen an der kirchenkritischen Presse zu vermuten. Auch innerhalb der Kirche gibt es zwei unterschiedlich informierte Öffentlichkeiten.

Im zweiten Modell nimmt die kirchliche Öffentlichkeit eine Diskrepanz in der Berichterstattung ihrer gruppeninternen Medien und der außerhalb der Kirche stehenden Medien wahr, im dritten Fall auch noch innerhalb dieser gruppeninternen Medien.

Welches der drei Modelle im untersuchten Fall für die tatsächliche Berichterstattung am ehesten zutraf, ist angesichts der Vorbemerkungen unschwer zu erraten. 


\section{Ergebnisse}

Im Untersuchungszeitraum von Juli 1989 bis Dezember 1992 entsprachen in den ausgewählten Zeitungen insgesamt 591 Artikel und Leserbriefe den Auswahlkriterien für die Inhaltsanalyse. Dabei veröffentlichten die katholischen Zeitungen und das "Publik-Forum" erwartungsgemäß deutlich mehr Artikel als das „DAS” und die beiden säkularen Zeitschriften.

Auffällig war die große Zahl von Leserbriefen in den katholischen Zeitungen, die bei "Dom" und „Glaube und Leben“ zwei Drittel, beim RM sogar drei Viertel aller Beiträge ausmachten, weiterhin die vielen Leserbriefe zur "Rolle der Medien im Konflikt" in der Paderborner Kirchenzeitung, in denen sich die Leser zur Rolle der (kirchenfernen) Presse negativ äußerten. Bereits dieser Befund spricht dafür, daß die Leser der katholischen Zeitungen offenbar eine Diskrepanz zwischen (Inhalt und/oder Intensität) der Berichterstattung in diesen Zeitungen und dem, was sie in den säkularen Medien beobachten konnten, wahrnahmen. Die Wahrnehmung dieser Diskrepanz durch die Leser motivierte sie zum Schreiben von Leserbriefen. Daß dies tatsächlich eine zutreffende Einschätzung sein kann, zeigte auch der Inhalt der Leserbriefe. Das Modell der "kirchenkonformen Berichterstattung" würde eine solche Diskrepanz und ihre Auswirkungen nicht erklären.

Neben diesen mehr formalen Beobachtungen sind aber die inhaltlichen Ergebnisse bezüglich der Gewichtung der Themenstrukturen von größerer Bedeutung. Insgesamt konnten 46 Themen verschlüsselt werden, die in die drei Bereiche "kirchliche Themen" (z.B. zum Zustand der Kirche allgemein, zu Reformen oder zu Autorität/Macht in der Kirche), „theologische Themen" (z.B. zu Angst und Erlösung, Sünde und Schuld, exegetischen Methoden oder moraltheologischen Fragen) und "konfliktgeladene Themen" (z.B. zum Verlauf des Konflikts und zum Verhalten der Beteiligten) unterteilt waren. Auch die Bewertungen von Themen, Personen und Ereignissen im Zusammenhang mit dem Konflikt wurden erfaßt.

Die Berichterstattung über den Konflikt, so sind die Ergebnisse bezüglich der Themenstrukturen zu deuten, entwickelte sich im Verlauf der Auseinandersetzung weg vom Modell zwei, einer unterschiedlichen Berichterstattung in allen Zeitungen, hin zu Modell drei, in dem sich auf der einen Seite kirchenferne und kirchenkritische Zeitungen in ihrer Berichterstattung einander annähern und sich auf der anderen Seite die kirchlichen bzw. katholischen Zeitungen einander angleichen.

Diese Daten lassen sich dahingehend interpretieren, daß die Kirchenzeitungen offenbar für die Berichterstattung anderer Medien keine Orientierungsfunktion (mehr?) besitzen, ganz im Gegensatz etwa zur Zeitschrift „Publik-Forum". Am deutlichsten zeigt sich dies, wenn man die Übereinstimmungen in der Struktur der Berichterstattung zwischen den verschiedenen Zeitungen im Zeitverlauf vergleicht. 
Es wird deutlich, daß gegen Ende des Konflikts die größten Übereinstimmungen in der Berichterstattung zwischen "Spiegel", "Zeit" und "Publik-Forum " bestanden, und zwar sowohl in der Betonung der Konfliktebenen als auch in der Auswahl der Themen. Die katholischen Zeitungen unterschieden sich in der Berichterstattung umso deutlicher von dieser Dreiergruppe, je weiter der Konflikt fortschritt. Während sich die katholischen Zeitungen im Verlauf des Konflikts immer weniger für die theologischen Themen des Konflikts interessierten (sondern für die Themen, die den Konflikt, den "Streit" der beiden Kontrahenten in den Vordergrund stellten), blieb diese Ebene für „Publik-Forum“ über den gesamten Konfliktverlauf hin gleich wichtig. Und die wichtigste Feststellung: Der "Spiegel" und die "Zeit" übernahmen diese Sichtweise des Konflikts. Deutlich macht dies auch ein statistischer Vergleich der Themenstrukturen der Zeitungen. Zu Beginn des Konflikts zeigen sich nur wenige Übereinstimmungen in der Rangfolge der wichtigsten Themen. Eine geringe Ähnlichkeit kann man in den letzten drei Monaten des Jahres 1989 allenfalls zwischen RM und "Dom" auf der einen Seite $\left(.43^{*}\right)^{6}$ sowie "Spiegel" und DAS auf der anderen Seite (.65 *) feststellen. Im Zeitverlauf zeigt sich dann eine interessante Veränderung: Gegen „Ende” des Konflikts im ersten Quartal des Jahres 1992 zeigen die katholischen Zeitungen die größten Ähnlichkeiten zu allen Zeitungen mit Ausnahme von "Spiegel" und "Zeit ". Diese beiden Zeitungen ähneln nun in der Rangfolge ihrer wichtigsten Themen innerhalb des Konflikts wesentlich mehr dem „Publik-Forum“ und dem DAS (Tabelle 1). Die Vermutung, daß sich gegen Ende des Konflikts die Berichterstattung des „Publik-Forum" bei den säkularen Medien durchsetzt, wird durch diese Beobachtung gestärkt.

Glaube

$\begin{array}{llllllll}\text { u. Leben } & .80^{* *} & & & & & \\ \text { Publik- } & & & & & & \\ \text { Forum } & .65^{* *} & .51^{*} & & & & \\ \text { DAS } & .68^{* *} & .42^{*} & .71^{* *} & & & \\ \text { RM } & .84^{* *} & .77^{* *} & .49^{*} & .57^{*} & & \\ \text { Zeit } & .38 & .42 & .50^{* *} & .54^{*} & .30 & \\ \text { Spiegel } & .40^{*} & .34^{*} & .51^{*} & .51^{*} & .34^{*} & .23 \\ & \text { Dom } & \begin{array}{l}\text { Glaube Publik- } \\ \text { u.Leben Forum }\end{array} & & \text { DAS } & \text { RM } & \text { Zeit }\end{array}$

Tab. 1: Ähnlichkeit der Themenrangfolge im I. Quartal 1992 (Spearman's Rho)

6 Der Koeffizient "Spearman's Rho" gibt die Übereinstimmung verschiedener Rangfolgen an. Der Wert +1 bedeutet völlige Übereinstimmung der Rangfolgen, der Wert -1 völlige Verschiedenheit, 0 bedeutet, daß keinerlei Korrelation besteht. Die mit "* " bezeichneten Korrelationen sind signifikant, solche die mit "**" gekennzeichnet sind, hochsignifikant. 


\section{Folgerungen}

Denn wenn man davon ausgeht, daß ein "framing" stattgefunden hat, spielten dabei Zeitungen wie „Publik-Forum“ eine entscheidende Rolle. Um einem Einwand gleich vorzubeugen: Die statistischen Zusammenhänge belegen natürlich keine kausalen Abhängigkeiten und alle daraus abgeleiteten Folgerungen bleiben hypothetische Konstrukte. Und auch die Korrelationen können aufgrund teilweise nur geringer Fallzahlen eher als Hinweise denn als Belege dienen. Erscheint es aber nicht naheliegend, daß die Redakteure des "Spiegel“ und der "Zeit " bei der Recherche für ihre Artikel auch die Kirchenzeitungen und das "Publik-Forum" zur Hand nahmen, um sich über den Streit zu informieren und sich ein Bild von der Stimmungslage in der Kirche zu machen? Und daß sie sich dann dafür entschieden, der Betrachtungsweise dieser Zeitungen mehr oder weniger zuzustimmen bzw. ihnen eine Sachkompetenz zuzusprechen oder nicht? $\mathrm{Ob}$ diese Entscheidung eher bewußt oder unbewußt geschah, in jedem Fall ist sie für die kirchlichen Zeitungen im untersuchten Fall ungünstig ausgefallen. Verfolgt man diesen Gedanken konsequent weiter, so werden die Kirchenzeitungen wohl auch schon bald nicht mehr zu den Medien gehören, die Journalisten säkular orientierter Medien bei ihrer Arbeit benutzen.

Offenbar haben die Katholische Kirche und ihre Medien an Glaubwürdigkeit verloren, sowohl bei den Lesern (die Auflagenzahlen müssen hier nicht kommentiert werden), als auch, was noch wesentlich weitergehende Konsequenzen hat, bei den Vertretern der säkularen Medien. Das ist im untersuchten Fall auch darauf zurückzuführen, daß die kirchlichen Zeitungen überraschenderweise nicht dazu in der Lage waren, über den Konflikt zwischen Drewermann und Degenhardt vor einem theologischen Horizont zu berichten, sondern im Laufe der Auseinandersetzung immer mehr Themen wie das Verhalten Drewermanns oder nicht-kirchlicher Medien in den Vordergrund ihrer Berichterstattung stellten. Die eigentliche theologische Auseinandersetzung spielte sich in Zeitungen wie dem "Publik-Forum" oder dem evangelischen "Deutschen Allgemeinen Sonntagsblatt" ab. Diese Tatsache korrespondiert mit dem im Zusammenhang mit dem Konflikt um Drewermann von Bischof Lehmann geäußerten Vorwurf, daß die Theologen angesichts des Konflikts versagt hätten: Nicht nur die Theologen, auch die kirchlichen Redakteure hätten sich anders verhalten können.

Die Kirchenzeitungen (und teilweise wohl auch der "Rheinische Merkur") stehen so tatsächlich in der Gefahr, immer stärker zu einer Art "Vereinszeitung" zu werden, die außerhalb kirchlicher Kreise, weder direkt in der Gesellschaft noch indirekt über die Meinungsführermedien, keine Beachtung mehr findet. Dagegen erfährt das „Publik-Forum “ eine Aufwertung, die man nicht allein mit einer allgemein kirchenkritischer werdenden Einstellung der Öffentlichkeit erklären kann. Eher 
scheint es so zu sein, daß die große Abhängigkeit der kirchlichen Zeitungen von der Amtskirche immer stärker als Widerspruch zu einem unabhängigem Journalismus gesehen wird - ob die "Schere im Kopf" des Kirchenredakteurs nun existiert oder nur vermutet wird.

Ein weiterer Gesichtspunkt kommt hinzu. Bei einer Analyse der Berichterstattung über den Drewermann-Konflikt fällt auf, daß die Kirchenzeitungen erst relativ spät ("Der Dom") bzw. anfangs nur in geringem Umfang („Glaube und Leben“) über den Konflikt berichteten. Soll man dies als einen Versuch werten, den Konflikt in seiner Anfangsphase einfach zu übergehen, ihn „totzuschweigen"? Ähnliches stellt ja auch die Studie von Kepplinger/Ehmig fest, wo die Tendenz festgestellt wird, "daß die Bistumspresse einer Diskussion, die ihr von anderen Medien aufgezwungen werden soll, weitgehend ausweicht ${ }^{\prime \prime}$. Erst durch die große Zahl von Leserbriefen konnten die Redaktionen der Kirchenzeitungen offenbar dazu gebracht werden, sich dem Thema in der ihm angemessenen Ausführlichkeit zu widmen. Diese Beobachtung läßt darauf schließen, daß sich die Kirchenzeitungen mit gewissen Eigengesetzlichkeiten der Massenmedien, hier etwa dem offensiven Zugehen auf Konflikte, noch nicht so recht anfreunden konnten. "Fehler, Fehlentwicklungen, Skandale aufzudecken", so schreibt Jürgen Hoeren, "ist letztlich Dienst an der Kirche - wird aber von dieser eher als lästige Kritik empfunden. ${ }^{\mu 8}$ Wenn die Kirchenzeitungen nicht einmal in einem Fall, der sie in vielerlei Weise selbst zutiefst betrifft, die offensive Auseinandersetzung wagen, werden sie es auch nicht bei anderen Themen, tun. Und diesen Eindruck gewinnen ja Kepplinger / Ehmig offenbar auch in ihrer Untersuchung, wenn sie vielfach das Fehlen einer eigenständigen, christlichen Betrachtungsweise bemängeln, durch die die Kirchenzeitungen an Profil gewinnen könnten.9

Wenn die Kirche mit ihren Kirchenzeitungen in der Zukunft noch eine Rolle bei der öffentlichen Meinungsbildung spielen will, erscheinen zumindest organisatorische Reformen unvermeidlich, die deutlich machen, daß es der Kirche mit ihren Bistumszeitungen nicht nur um einen Verlautbarungsjournalismus geht. Denn diejenigen Kritiker, die in den Kirchenzeitungen nicht zu Wort kommen, werden dazu gezwungen, sich ein Forum in den kirchenkritischen und säkularen Medien zu suchen. Verbunden mit solchen Reformen erscheint aber auch ein radikaler Reflexions- und Orientierungsprozeß $B$ über die Funktion kirchlicher Medien als dringend notwendig, damit die kirchlichen Medien nicht von den Entwicklungen in einer immer stärker massenmedial orientierten Gesellschaft überrollt werden.

7 Vgl. H. M. Kepplinger/S. Chr. Ehmig, Form und Inhalt (Anm. 1), 14.

8 J. Hoeren, Ethos und Angst. Das Verhältnis der Amtskirche zur Öffentlichkeit, in: Diakonia 22 (1991) 309-316, 316.

$9 \quad$ Vgl. H. M. Kepplinger/S. Chr. Ehmig, Form und Inhalt (Anm. 1), 19. 


\section{Deutschlands "fromme" Presse \\ Zwischenbilanz katholischer Chefredakteure}

Unter dem Titel "Deutschlands fromme Presse" haben Günther Mees und Ferdinand Oertel eine ,Zwischenbilanz katholischer Publizisten' als Buch im Knecht-Verlag, Frankfurt (232 S., mit zahlreichen Fotos, geb. DM 48,-) herausgegeben. Darin berichten 17 Mitarbeiter der Kirchenpresse, die jetzt in den Ruhestand getreten sind, über ihre Erfahrungen in der Zeit nach dem Zweiten Vatikanischen Konzil. Wir veröffentlichen mit freundlicher Genehmigung des Knecht-Verlages einige Auszüge, in denen die Autoren ein Resümee ihrer Tätigkeit ziehen. Diese Auszüge bilden gleichzeitig einen Beitrag "zur Debatte" über die Zukunft der Kirchenzeitungen.

\section{Zeitgemäße Antwort steht aus}

Konkretisiert man die Diskussionspunkte der vergangenen 25 Jahre, dann stößt man auf Formulierungen, die offenbar auch heute noch einer zeitgemäßen Antwort im weitgefaßten Raum der "frommen Presse" bedürfen:

Konzentration in der Kirchenpresse - Kirchenpresse als Dialogforum oder "zweite Kanzel“ - Organ der Amtskirche oder aller Katholiken - Werkzeug der Seelsorge oder Medium eines eigenständigen Kultursachbereichs - Publizistische Nachwuchsförderung und Ansprache neuer junger Leserschichten - Pfarrblätter und Bistumszeitungen Zusammenarbeit mit der Gesellschaft Katholischer Publizisten (GKP) und im weiteren Sinne mit weltlichen publizistischen Einrichtungen.

Der lebhafte Dialog in der "frommen Presse" Deutschlands ist Ausdruck auch einer manchmal kräftezehrenden Vitalität. Wer sich diesem Dialog verweigert, stellt sich aber einem notwendigen Neuanfang in den Weg.

Günther Mees

\section{Alte Tabus brechen}

Die 1994 im Auftrag der MDG vom Institut für Demoskopie in Allensbach erstellte Studie "Chancen für die Bistumspresse" weist nach, daß in breiten Kreisen der katholischen Bevölkerung durchaus Interesse an kirchlichen Themen besteht: vorwiegend am Leben auf der Gemeindeebene und an weltkirchlichen Vorgängen. $\mathrm{Ob}$ es genügt, die bestehenden Bistumszeitungen in dieser Richtung weiterzuentwickeln (was notwendig ist, weil sie in einem festumrissenen Lesersegment ihren Platz haben), oder ob Neues gewagt werden muß, neue Objekte, 
die in ihrem eigenen Verständnis neu und neu im Verständnis potentieller Leser sind, das ist wohl eine Frage, die niemand im voraus beantworten kann. In jedem Fall müssen Tabus angegangen werden, die wir bei unserer Verwirklichung des nachkonziliaren Selbstverständnisses nicht "knacken“ konnten: daß jedes Bistum sein eigenes Blatt braucht (was bis zur zwangsweisen Einführung in der NS-Zeit auch nicht der Fall war), daß Kirchenzeitungen nur kirchliche Herausgeber haben müssen (und kein Joint-Venture mit säkularen Verlagen eingehen können), daß sie an bestimmte Verlage und Druckereien, Formate und Vertriebswege gebunden sind, - um nur einige zu nennen.

Aus den Aktivitäten der mit der Kirchenpresse befaßten Bischöfe und kirchlichen Institutionen der letzten Jahre möchte ich nicht ganz ohne Hoffnung schließen, daß 25 Jahre nach dem Erscheinen von "Communio et progressio" neue Perspektiven erschlossen werden sollen.

Ferdinand Oertel

\section{Mut beweisen}

... wie es scheint, fährt der Zug (der Kooperation - die Red.) wieder zurück. Letzte Reste von Gemeinsamkeit schmelzen dahin. Eine überwunden geglaubte Ortskirchen-Ideologie feiert Auferstehung. Dem Aggiornamento des Konzilspapstes Johannes XXIII. wird der Ruf nach der Rückkehr hinter die Mauern entgegengestellt. Statt dem Mut zur Weite des Denkens herrscht zunehmend die Angst vor Verunsicherung der verbliebenen Herde.

Diese Trends sind einem selbstbewußten Journalismus in der Kirche nicht gerade förderlich. Mit der Abhängigkeit von Zuschüssen schwindet zudem der "Mut vor Königsthronen". Und das wäre fatal. Fatal für die Kirchenpresse, aber auch fatal für die Kirche selbst. Das sogenannte Kirchenvolksbegehren hat gezeigt, daß es nicht kleine Zirkel sind, die aufbegehren, sondern daß der Unmut am gegenwärtigen Zustand der Kirche bei uns weitverbreitet ist. Und darüber muß geredet, muß geschrieben werden. Mit Mut zur eigenen Meinung und nicht mit Verstecken hinter sogenannten "großen" Namen. Oder mit Rückzug in die Wagenburg.

Dabei bleiben auch in Zukunft die Probleme bestehen, die schon unserer Generation zu schaffen gemacht haben: Es bleibt die Schwierigkeit, in dieser lauten Welt der Sensationen und Skandale "gute", positive Nachrichten zu verbreiten. Es bleibt die Diskrepanz zwischen der abgehobenen Sprache kirchlicher Verlautbarungen und der Umgangssprache unserer Zeit. Es bleibt auch der Erwartungsdruck der kirchlichen Herausgeber an die Kirchenpresse, all das leisten zu sollen, was Religionsunterricht und Predigt, Hirtenbriefe und Enzykliken nicht 
schaffen: die Neuevangelisierung der Menschen. Es bleibt auch der Spagat zwischen den Lesern, die Sehnsucht nach "früher" haben, und denen, denen es nicht schnell genug mit dem Fortschritt geht.

Und es bleibt der Auftrag der Kirche, die ganze Welt „heimzuholen" und nicht nur eine auserwählte Schar. Den Journalisten in der Kirche bleibt die Aufgabe, immer wieder daran zu erinnern und sich gegen Tendenzen zur Selbstgenügsamkeit und Selbstzufriedenheit zu stellen.

Hermann Josef Kreitmeir

\section{Es geht um zentrale religiöse Fragen}

Der moderne Mensch hat sich aus vielfältigen Gründen von Kirche und Glauben entfernt, aber die zentralen religiösen Fragen wiegen ohne Zweifel am schwersten. Wer heute ein Kirchenblatt bezieht, will sich in ihm mit diesen Fragen wiederfinden, ist mit den Antworten der Tradition nicht zufrieden. Es mag sein, daß eine gewisse Leserschicht gerade auf diese Antworten pocht (solche Leute also, die schon vorher zu wissen meinen, was "richtig" glauben heißt), aber wir wissen alle, wie stark diese Form von Gläubigkeit in den letzten fünfzig Jahren zusammengeschmolzen ist. Die Generationen des dritten Jahrtausends nach Christus werden die Substanz der Lehre Christi anders annehmen und $\mathrm{zu}$ leben versuchen, als dies die Jahrhunderte vorher taten. Diese Leserinnen und Leser von morgen müssen an unsere Presse gebunden werden.

Das heißt nicht, daß nun alle anderen Fragen, die zur Zeit im deutschen Katholizismus Priorität genießen, ganz zu verschwinden hätten, jene sekundären Probleme, die der Geist der Zeit auch hat und die ohne Zweifel wichtig sind. Aber weder die Erlaubnis zur Priesterheirat und das Frauenpriestertum noch die Demokratisierung der kirchlichen Strukturen und menschliche Erleichterungen in der (Sexual-)Moral der Kirche werden uns den verlorenen christlichen Glauben breiter Schichten von Menschen wiederbringen. Die wirklichen Entscheidungen über die Christlichkeit unseres Kontinents fallen dort, wo es um Gott selbst - sein Bild und sein Wirken in dieser Welt - geht; dort, wo der historische Jesus und der Christus des Glaubens nicht mehr richtig zusammenkommen; dort, wo moderne Wissenschaft Weltgeschichte schreibt und sie nicht weiß, wo sie in ihr die Heilsgeschichte unterbringen soll. Das sind Fragen, die in den Diskurs der Kirchenpresse gehören, wenn sie denn eine solche sein will.

Manfred Plate 


\section{Erkenntnisse vorhanden}

Inzwischen sinken die Auflagen weiter, und die Diskussion über Auftrag, Krise und Bedeutung der Kirchenpresse hält unvermindert an. Währenddessen gibt es im Markt der Publikumszeitschriften Monat für Monat Innovationen und publizistische Wagnisse. Öffentliche Diskussionen über den öffentlichen Auftrag dieser Presse gibt es selten.

Nicht alles, was sich die MDG bei ihrem Beginn 1975 vorgenommen hat, ließ sich verwirklichen. Sichtbare Erfolge, wie Auflagensteigerungen, Schaffung großer leistungsfähiger wirtschaftlicher Einheiten sind nur bedingt gelungen. Die optische und inhaltliche Qualität der Blätter hat sich weiterentwickelt. Das wirtschaftliche Bewußtsein in den Verlagen hat zugenommen. Die Zahl der katholischen Zeitschriften blieb weitgehend konstant. Die Ausbildungsprogramme haben sich bewährt. Und die MDG als Unternehmensberatung im konfessionellen Bereich ist anerkannt.

Die Idee, aus 22 einzelnen Kirchenzeitungen große regionale Magazine zu entwickeln, hat sich bisher als nicht machbar erwiesen. Heute können die einzelnen Blätter mit der rein äußerlichen Qualität der Medienkonkurrenz an den Kiosken nicht mehr Schritt halten. Auch ihre Inhalte werden oft von der bunten Lebensfreude übertroffen, die in säkularen Medien gang und gäbe ist. "Klein, unwichtig, grau?", so titelte am 16. Februar 1996 "Die Zeit“ in einem Beitrag über die konfessionelle Presse. Hat sie recht?

Ich weiß, daß es zunehmend schwerer wird, die Anliegen der Kirche auf dem "Markt" gegen Gebühr anzubieten. Mit Sparmaßnahmnen allein ist es nicht mehr getan, um einzelne Kirchenzeitungen zu erhalten. Man kann Objekte durch straffes Kostenmanagement auch so "austrocknen“, daß sie für den Leser uninteressant werden.

Dabei ist das alles nicht neu. Wege zur "Gesundung" wurden durch die MDG immer wieder aufgezeigt. Mit ih:er Hilfe gab es in den vergangenen zwanzig Jahren immer wieder aufschlußreiche Leserbefragungen (Copytests). 1987 wurde eine Funktionsanalyse und 1994 schließlich eine groß angelegte Allensbacher Befragung durchgeführt. Es sind also genügend Erkenntnisse und fachlich fundierte Empfehlungen für den Weg in die Zukunft vorhanden. Es; wird entscheidend sein, sie jetzt konsequent und gemeinsam umzusetzen. Die Zeit der vielfältigen Grundsatzdiskussionen und subventionierter Eigenbröteleien sollte zu Ende gehen. Es ist zu hoffen, daß dies mit Hilfe der MDG auch künftig gelingt.

Raimund Brehm 


\section{Veränderungen notwendig und möglich}

Formale Änderungen am Layout sind für die Zukunft notwendig, aber sekundär. Zunächst kommt die thematische und inhaltiche Neuorientierung und dann in ergänzender und dienender Funktion das Layout. Farbe und Layout dürfen nicht der Ansatz der Reform sein. Dies wäre nur Kosmetik.

Wenn Herausgeber, Verleger und Redaktionen sich vor der thematischen und inhaltlichen Neurorientierung nicht scheuen, vor der Änderung der eigenen Einstellungen aufgrund der Erkenntnisse der Marktanaiyse nicht zurückschrecken, dann gibt es keinen Zweifel; dann haben die 4,9 Mio. des weitesten Leserkreises und die 1,2 Mio. Abonnenten Bestand und die 31 Prozent des sogenannten psychologischen Potentials eine Chance, hinzugewonnen werden zu können.

Bevor dieser Zukunftschancen nicht durch harte Arbeit und kluge Entscheidungen und die notwendigen personellen und finanziellen Mittel gegeben sind, sind vorschnelle Entscheidungen - aus welchen Gründen auch immer -, die Bistumszeitung einzustellen oder durch kostenlose Verteilblätter zu ersetzen, nicht nur Flucht vor der Verantwortung, sondern auch intellektuell unredlich, weil die Chancen für die Zukunft der Bistumszeitungen aufgrund empirischer Untersuchungen, analytischer Daten und ausreichender Interpretationshilfen für jeden Verantwortlichen erkennbar sind.

Den geringen Auflagen einzelner Bistumszeitungen, den hohen Qualitätsanforderungen, den hier und dort notwendigen personellen Aufstockungen stehen zurückgehende Finanzen der Bistümer gegenüber. Das macht mindestens jetzt die von weitsichtigen Leuten schon seit Jahrzehnten geforderten Kooperationen, ja Fusionen von Bistumszeitungen zwingend nötig. Die Anforderungen an die Redaktionen könnten durch gemeinsame überregionale Teile mehrerer Kirchenzeitungen erfüllt werden, und die freien Kapazitäten könnten für eine Verbesserung der Bistumsberichterstattung genutzt werden, von der Einsparung durch gemeinsame Produktion, gemeinsamen Vertrieb, gemeinsame Werbung und Administration ganz zu schweigen. Die Umfrage zeigt eindeutig positive Meinungen zu diesem Punkt. Herausgeber, Verleger und Leser halten eine Kooperation mehrerer Bistumsblätter für richtig. Eine wichtige Chance der Bistumszeitungen liegt also auch in der Bereitschaft zur strukturellen und administrativen Veränderung.

Heikn Klinge 